1

2

3

\title{
Stochastic Network Models for Logistics Planning in Disaster Relief
}

\author{
Douglas Alem* \\ Production Engineering Department \\ Federal University of São Carlos, Sorocaba, Brazil. \\ Alistair Clark \\ Department of Engineering Design and Mathematics \\ University of the West of England, Bristol, BS16 1QY, United Kingdom. \\ Alfredo Moreno \\ Production Engineering Department \\ Federal University of São Carlos, Sorocaba, Brazil. \\ * Corresponding author: \\ E-mail address: douglas@ufscar.br . \\ Phone number: +55 1532297425
}

\begin{abstract}
Emergency logistics in disasters is fraught with planning and operational challenges, such as uncertainty about the exact nature and magnitude of the disaster, a lack of reliable information about the location and needs of victims, possible random supplies and donations, precarious transport links, scarcity of resources, and so on. This paper develops a new two-stage stochastic network flow model to help decide how to rapidly supply humanitarian aid to victims of a disaster within this context. The model takes into account practical characteristics that have been neglected by the literature so far, such as budget allocation, fleet sizing of multiple types of vehicles, procurement, and varying lead times over a dynamic multiperiod horizon. Attempting to improve demand fulfillment policy, we present some extensions of the model via state-of-art risk measures, such as semideviation and conditional value-at-risk. A simple two-phase heuristic to solve the problem within a reasonable amount of computing time is also suggested. Numerical tests based on the floods and landslides in Rio de Janeiro state, Brazil, show that the model can help plan and organise relief to provide good service levels in most scenarios, and how this depends on the type of disaster and resources. Moreover, we demonstrate that our heuristic performs well for real and random instances.
\end{abstract}

Keywords: OR in disaster relief; Humanitarian logistics; Emergency logistics planning; Two-stage stochastic programming; Risk-aversion. 


\section{Introduction}

Floods and landslides are just two of the various types of disaster that plague Brazil year after year, leaving a trail of huge destruction. According to Valencio (2014), the problem of disasters in Brazil should not be considered residual; it suffices to analyse the number of municipalities which declared an emergency situation or state of public calamity between the years 2003 and 2013 to confirm that disasters are, in fact, recurrent events. During this period, an average of 1,368 municipalities, about $24 \%$ of Brazilian municipalities, had some sort of socioeconomic problem as a result of environmental disasters - from droughts in the northeast to flooding in the south region. In the same line, Yodmani (2001) argues that disasters should not be seen as simply extreme events caused only by natural forces, but also as symptoms of under-development in countries.

In July 2013, the Civil Defense of Brazil and its Postal Service signed a contract for the prepositioning of strategic stocks for humanitarian assistance in certain regions of the country in an attempt to mitigate disaster impacts (Presidency of the Republic, Brazilian Government, 2013). The main idea behind such a policy is to provide a faster response, possibly by decreasing lead times between the request and arrival of supplies. For this purpose, though, an optimized system is needed to cross-check information about safety stocks (location, availability, etc) with updated information about the disaster (e.g., affected areas, number and need of the victims, road damage) and to determine the quantity and sizes of existing vehicles required to deliver supplies. Such prepositioning, without mentioning the provision of emergency relief and supplies to disaster victims, is fraught with planning and operational challenges (Balcik et al, 2010) mostly due to the scarcity of overall resources and the inherent uncertainties of disasters.

Thus, in order to respond to disasters effectively, governments and humanitarian organisations need to consider these issues when designing effective relief supply chains. However, Gonçalves (2011) showed that humanitarian decision makers often make non-optimal decisions in the field by over-reliance on past experience, over-confidence in their own unaided decision-making abilities, and the use of simple decision heuristics. The resulting poor decisions motivate this paper to explore stochastic and dynamic models that can handle the urgent complexity and uncertainty faced by decisions makers. In particular, we propose an optimization model that could be used within a decision-aid tool by the Brazilian agencies that are currently responsible for providing rapid responses in disaster relief. Our model encompasses common decisions regarding logistics planning, such as how to dispatch emergency aid from warehouses to affected areas over a number of time periods in order to alleviate suffering during relief operations, that is, to decide the most suitable routes, which vehicles should be used, in which periods and in which quantity, and so on.

The model also balances the immediate lack of external supplies by allowing (i) the procurement of emergency aid during a response; and (ii) the prepositioning of emergency aid before a disaster strikes. The first option is based on Brazilian humanitarian practices where procurement usually occurs some days or more after the start of the humanitarian operations in the same area where the disaster struck. However, only a few emergency aid items are likely to be available, in very limited amounts and at a very high cost, since local vendors often take advantage of the vulnerable situation. The second option may be seen as a global trend to overcome the suddenness of disasters (Salmerón and Apte, 2010; Duran et al, 2011; Jr. et al, 2012; Davis et al, 2013). Additional decisions supported by the proposed model concern 
defining appropriate inventory levels and budget allocation among the various operations. Backlogging is permitted at a very high cost, as we know that in practical problems there are not enough resources to meet the demand from all victims.

For Oztaysi et al (2013), uncertainty is a crucial challenge that must be overcome in disaster management. In fact, immediately after or during a natural disaster, the number of affected people (homeless, displaced, injured, deaths) may be only vaguely known and recipient demand will be difficult to predict in terms of timing, location, type, and size. Suddenly-occurring large demand and short lead times for a wide variety of supplies will contrast with periods of low demand (Kovács and Spens, 2009; Sarkis et al, 2010). In addition, since the situation is often unclear in the immediate aftermath of the disaster, some routes might be total or partially blocked, with uncertain information about any damage. In the megadisaster of the Serrana region of Rio de Janeiro (Brazil) in 2011, 14 state highways from 7 different cities suffered traffic blockages due to landslides and flooding, besides the countless number of urban and rural access roads that were also damaged. Although many emergency aid supplies can be strategically prepositioned, only some of them will remain usable, depending on the impact and location of the disaster.

Supplies and monetary funds may also be affected by the degree of the disaster. Recent studies suggest that donor behaviour may depend not only on measurable parameters - such as an aid agency's use of donated funds, fund-raising cost factors, and donors' unit utility of donations (Toyasaki and Wakolbinger, 2011) - but also on subjective factors that influence the decision to donate, such as how people perceive a certain disaster (Zagefka et al, 2011), and the role of the media, social networks, etc. (Brito Junior et al, 2013). As supplies and monetary budgets usually come from a variety of organisations, they are considered to have uncertain values as well. This uncertainty is further complicated by the phenomenon of material convergence (Fritz and Mathewson, 1957; Holguín-Veras et al, 2012) whereby well-meaning non-professional donors supply huge quantities of unsolicited goods of doubtful usefulness that can (and often do) clog the supply chain, disrupting the flow of urgent aid to recipients in need.

In this paper, uncertainties are modelled via scenario-based two-stage stochastic programming. The first-stage plans the prepositioning of emergency aid and the overall capacity of each type of vehicle (fleetsizing). The second-stage response makes operational decisions for transportation - including the detailed decision regarding the assignment of vehicles to routes - as well as inventory, shortages, and procurement. The objective function is flexible, being able to minimise total expected costs or prioritize the meeting of recipient demand while avoiding excessive inventory. We also show how that the stochastic programming model can be straightforward extended to consider different risk-averse preferences to produce less risky or reliable solutions and/or to improve fairness.

As the mixed-integer stochastic programming models are huge and computationally challenging even for small-sized instances, we develop a two-phase heuristic that performs very well for real and random instances. The first phase solves a (smaller) simplified version of the model that overestimates the overall number of vehicles in the first-stage. The second phase then fixes the first-phase decisions regarding the operational distribution flow and re-solves the original model to determine the remaining decision variables.

Our overall numerical results have important implications in terms of roles within the humanitarian 
supply chain in efficiently managing both the preparedness and response phases of most sudden-onset disasters.

This paper is organised as follows. Section 2 reviews the most relevant literature. Section 3 develops the proposed stochastic optimization model. Section 4 shows how to extend the risk-neutral stochastic model to incorporate risk preferences. Section 5 presents a two-phase procedure to determine goodquality solutions within a reasonable amount of time. Section 6 implements the model on real data from the 2011 floods and landslides in Rio de Janeiro state, Brazil, and analyses the computational results. The paper concludes in section 7 with a discussion of the model's value and flags remaining challenges and opportunities for future research.

\section{Related Literature}

Possibly due to the increased frequency and severity of disasters around the world and to their greater visibility on television and social media in recent decades (Franks, 2013; Seib, 2013), these wrenching events have been the subject of growing public concern and research interest (Smith et al, 2009). This paper will not review all decision models for disaster management and emergencies, but rather will focus on the quantitative management of uncertainty in emergency logistics planning in disaster relief, considering only recent research on scenario-based stochastic programming approaches in transportation and distribution relief within network flows. The reader who is interested in wider surveys of the application of quantitative models and operational research to humanitarian logistics can consult Altay and Green (2006); Van Wassenhove (2006); Caunhye et al (2012); De la Torre et al (2012); Holguín-Veras et al (2012); Ortuño et al (2013); Galindo and Batta (2013); Leiras et al (2014) and Özdamar and Ertem (2015) amongst others.

Two-stage stochastic programming with recourse models have been successfully used in humanitarian logistics and disaster management as they allow the modeller to represent pre- and post-event phases together via first- and second-stage decision variables. Barbarosoğlu and Arda (2004) appears to be the pioneer paper that developed a two-stage stochastic model for both pre-event and post-event stages in disaster response, considering supply capacity and demand as random variables approximated by multiple scenarios. In addition, following a two-stage paradigm, but with different first- and second-stage decisions, Chang et al (2007) presented a scenario-based model adapted to represent a multi-echelon network in a flood disaster rescue system.

Relief resource allocation problems have also been researched in the following papers. Mete and Zabinsky (2010) designed a two-stage stochastic model to first locate possible medical storage centres and their stock levels before the disaster, then to deliver medical supplies to hospitals for each disaster scenario. Salmerón and Apte (2010) used a two-stage stochastic optimization model for the strategic positioning and sizing of a variety of relief resources, followed by scenario analysis to deploy the resources in the event of a disaster. Prepositioning decisions were also the focus of Rawls and Turnquist (2010), and are discussed in Rawls and Turnquist (2012), Noyan (2012), and Hong et al (2015). Rawls and Turnquist (2010) sought the pre-event prepositioning of relief supplies, using multiple scenarios to represent the huge amount of uncertainty regarding the date, location, and intensity of an eventual disaster and the difficulty of forecasting its impact on transport infrastructure. Noyan (2012) extended Rawls and Turnquist's model 
to include attitudes to risk by making use of the approach known as conditional-value-at-risk (CVaR), widely used in financial optimisation. Rawls and Turnquist (2012) developed a short-term planning model for pre-disaster use to meet uncertain needs in the first few days after the start of the disaster, requiring that a given high percentage of all scenarios (a type of $\alpha$-reliable constraint) should fully meet needs. Hong et al (2015) focused on location of distribution centers and positioning of emergency supplies in long-term pre-disaster planning in an attempt to improve network reliability. For this purpose, their paper developed a chance constrained model and proposed tractable reformulations to solve more efficiently problems with moderate-size networks and a large number of scenarios.

The last mile distribution in post-disasters was investigated by Noyan et al (2015). The authors followed the two-stage paradigm to define the location of points of distribution in the first-stage and to determine the amount of supplies delivered to them in the second-stage in an attempt to maximize the expected accessibility to the distribution centers. Two-stage programming with recourse was adopted to plan relief shelters and then the allocation of evacuees to shelters in Li et al (2011) in which the authors adopted a two-echelon network to represent both evacuee and resource flows to permanent and temporary shelters. In contrast, Döyen et al (2012) developed a two-echelon stochastic model for modelling flows between regional and local rescue centers, as well as locating them, prepositioning inventory, and then optimising cover and transport after the disaster, testing over many scenarios.

The integration between location and routing in emergency logistics is also tackled in Rennemo et al (2014) and Ahmadi et al (2015). The former authors have developed a first multi-stage stochastic programming model to deal with a facility routing model in humanitarian logistics. Their three-stage model incorporates the opening of local distribution facilities in the first-stage, the initial allocation of supplies in the second-stage, and the last mile distribution of emergency aid in the third-stage. The authors illustrated the outperformance of the model in comparison to deterministic approaches via the evaluation of both the expected value of perfect information and the value of stochastic solution. Ahmadi et al (2015) proposed a location-routing model under network failure, multiple uses of vehicles, and standard relief time. Network failure is modelled via a two-stage stochastic program with random travel time and analysed using a case study in the San Francisco district.

A different stochastic approach to deal with the issue of the imbalance between supply and demand in post-disaster emergency logistics operations was suggested by Sheu and Pan (2015). Stochastic dynamic programming was used to determine the amount and the type of emergency resources to the affected areas. The authors pointed out the importance of collaboration among relief suppliers to alleviate the impact of an imbalanced relief supply chain through a number of numerical experiments.

To take advantage of the temporal nature of the disaster, Rottkemper et al (2012) developed a rollinghorizon transshipment and inventory relocation model to reallocate goods as a disaster unfolds without prejudicing ongoing relief operations, resolving the model repeatedly for each time period, and updating as the situation changed over time.

Only a few authors have incorporated robustness issues within humanitarian operations. BozorgiAmiri et al (2013) considered multiple sources of uncertainty, including possible damage to prepositioned supplies, applied to earthquake scenarios in Iran. The authors used so-called robust optimization in the sense of Mulvey et al (1995) to simultaneously reduce the variability of the second-stage costs and the 

in good but wasteful cover in worst-case scenarios, so that it provides a range of cover-waste tradeoffs to choose from. The $p$-reliable regret criterion used by the authors resembles the $\alpha$-reliable criterion in Rawls and Turnquist (2012).

Table 1 summarizes the literature review with respect to scenario-based stochastic programming network flow models in disaster relief and shows the main differences between the existing literature and this paper. Only a few papers consider dynamic or multiperiod settings, although most authors recognize a disaster as also being a temporal event. In fact, most papers that present dynamic models ignore their inherent uncertainties, e.g., Özdamar et al (2004). Note too that some practical characteristics have been neglected by the literature so far, such as time-varying lead times and multiple modes of transportation. Regarding the decisions supported by the model, budget allocation, fleet sizing and procurement are rarely jointly considered in the current literature. Even though many papers account for random demands, networks and capacities, it seems that random supply has only recently been considered and random budgets have never been studied before. Most authors ignore the potential disadvantages of using riskneutral approaches, i.e., very pessimistic worst-case solutions, or the high-variability of the second-stage decisions. In fact, the importance of analysing robust or risk-averse policies in stochastic network models for disaster relief has been pointed out only recently by Noyan (2012). Most papers develop solution procedures in order to obtain fast good-quality solutions. Exact and heuristic methods are usually based on decomposition schemes in an attempt to produce smaller and easier solvable problems. Finally, note that flooding and landslides are only rarely studied by the specialised literature as most papers deal with hurricane and earthquake disasters in USA.

Specifically, our contribution in this article can be summarized as follows. Firstly, we propose a new stochastic model that combines important characteristics in terms of network structure, decisions and stochastic treatment. Although some articles have already studied various sources of uncertainty in similar contexts, we combine them to analyse their relationship. Moreover, we use a scale system to categorize historical data from flooding and landslide disasters in Rio de Janeiro State to generate plausible scenarios. Secondly, we extend the traditional risk-neutral model to consider risk-averse attitudes via state-of-art risk measures, namely, so-called minimax-regret, semideviation and CVaR. Thirdly, we develop a solution procedure that exploits a simplified model to construct a feasible solution and demonstrate its good performance in different instances.

[Insert Table 1 here]

\section{A Two-stage Stochastic Network Model for Disaster Relief}

Our two-stage network flow model encompasses multiple types of emergency aid, multiple types of vehicles, multiple time periods, and multiple scenarios. The core of the model is the transport of emergency aid among nodes, but it is possible to adapt the model for the evacuation of people from affected areas to relief centers via simultaneous flows and counter-flows. Although the mathematical model is general enough to represent different disaster types, it is particularly suitable for help in logistics planning of sudden and localized onset disasters (Apte, 2009), due to its main characteristics in terms 

(the Red Cross, ONG's, etc.) can then flexibly use the model to represent their particular challenges.

\subsection{Assumptions and limitation of the model}

At first, nodes are partitioned only into two subsets: nodes with and without demand. We assume that nodes with demand represent relief center points and that nodes without demand represent warehouses points where emergency aid will be prepositioned. In addition, prepositioning is permitted only in the first time period, in an attempt to represent its strategic allocation well before a disaster strikes. Unused prepositioned emergency aid in the first time period becomes regular inventory afterwards. Procurement of emergency aid is allowed in all periods, but only at relief center points.

The optimal distribution of emergency aid among nodes is defined by their flow along each route (a network arc between two nodes) and by determining a suitable size of transport fleet, i.e., the quantity of each type of vehicle necessary to carry the emergency aid, considering both weight and volume loading capacities. The model is formulated assuming there is just one connection from one node to another for each type of vehicle - if not then dummy intermediate nodes can be inserted to represent different connections. There is a maximum amount of emergency aid that can be prepositioned and procured, and a maximum available number of each type of vehicles that can be hired in the first stage.

The model attempts to minimise clogging within the supply chain due to material convergence (Holguín-Veras et al, 2012) by pulling aid through as a function of specific recipient needs rather than pushing supplies into the chain. However, the elimination of unneeded donated goods at prepositioning warehouses remains a modelling challenge that is beyond the scope of this paper.

The monetary budget may be used for transportation and procurement of emergency aid; any unused budget in period $t$ is passed to period $t+1$ without interest.

To take uncertainty into account, we propose a scenario-based approach within a two-stage stochastic programming paradigm. We consider a probability space $(\Xi, \mathcal{F}, \Pi)$, where $\Xi$ is the set of realizations, $\mathcal{F}$ is the set of events $\mathcal{F} \subseteq \Xi$, and $\Pi$ is the probability measure. Associated with a particular realization $\xi \in \Xi$, there is a probability of occurrence $\pi^{\xi}$, such that $\pi^{\xi}>0$ and $\sum_{\xi} \pi^{\xi}=1$. In this paper, we also assume that the random variables become known only at a single moment after disaster strikes, thus not revealing themselves "by time". In addition, the random variables and decisions are not intertwined; our scenarios are independently sampled over the full horizon, justifying our choice for two-stage programming instead of a multistage version.

Before disaster strikes, we have to strategically define the quantity and location of prepositioned emergency aid, as well as determine the overall capacity of each type of vehicle to perform (in the "future") effective emergency logistics, since hiring a suitable fleet can be a very bureaucratic and time-consuming public bidding procedure. Thus both decisions are naturally considered as first-stage variables. After disaster strikes, we determine the second-stage decision variables based on the updated information on the quality of the routes, supplies, demands and donation. Those decisions include the flow of emergency aid among arcs, the number and type of vehicles necessary to perform distribution, procurement issues, inventory and backlogging. The first use of the second-stage model should be as soon as possible after the start of the disaster. Its use thereafter will be on a rolling-horizon basis with updated information 
and scenarios as the impact of the disaster becomes clearer and fresh resources become available. An overview of the model's structure and usage is depicted in Figure 1.

[Insert Figure 1 here]

Although the location of relief centers and warehouses may have an important impact in certain relief supply network, we assume that they are well located beforehand so we focus on prepositioning, distribution, fleet decisions, etc. In our application, we consider all possibilities of location of both warehouses and relief centers, since we consider one relief center for each affected area and one warehouse for each big city. Other model's limitations include its data requirements, both deterministic and stochastic. The latter in particular are often guestimates for a given scenario, as acknowledged in the computational tests of Section 6. Moreover, the identification of a representative set of scenarios requires knowledge and experience of past or similar disasters, while climate change may cause new scenarios to occur, for example, flooding on a scale not seen before in many regions (Arnell and Lloyd-Hughes, 2014). In addition, the risk-aversion extensions developed in section 4 take much longer to solve and tend to increase first-stage costs (but reduce the variability of second-stage costs) as shown and discussed in Section 6.

\subsection{Model Formulation}

The full mathematical model uses the following notation.

\section{Indices and Sets}

$\mathscr{I} \quad$ Set of types of emergency aid indexed by $i$ (food, water, medicine kits, etc.).

$\mathscr{R} \quad$ Set of types of vehicles indexed by $r$ (trucks, boats, helicopters, etc.).

$\mathscr{N}^{r c} \quad$ Subset of demand nodes, or relief center nodes, indexed by $n$ and $m$.

$\mathscr{N}^{w} \quad$ Subset of supply nodes, or warehouses, indexed by $n$ and $m$.

$\mathscr{N} \quad$ Set of all nodes, indexed by $n$ and $m\left(\mathscr{N}=\mathscr{N}^{r c} \cup \mathscr{N}^{w}\right)$.

$\mathscr{T} \quad$ Set of time periods, indexed by $t$.

$\Xi \quad$ Set of scenarios, indexed by $\xi$. 
$c_{r}^{\max } \quad$ Available number of vehicles of type $r$.

$c_{i}^{w}$ and $c_{i}^{v} \quad$ Weight and volume of emergency aid $i$, respectively.

$g_{r}^{w}$ and $g_{r}^{v} \quad$ Weight and volume capacities of vehicles of type $r$, respectively.

$\ell_{\text {rnmt }} \quad$ Lead time to transport any emergency aid by vehicles of type $r$ along arc $n \rightarrow m$ in period $t$.

$p_{i}^{\max } \quad$ Maximum amount of emergency aid $i$ that can be prepositioned.

$o_{i n}^{\max } \quad$ Maximum amount of emergency aid $i$ that can be procured at node $n$.

$\alpha_{r n m} \quad$ Unit shipping cost by vehicles of type $r$ through the arc $n \rightarrow m$.

$\beta_{r} \quad$ Unit rental cost for using vehicles of type $r$.

$\iota_{i n}^{+} \quad$ Unit holding cost for emergency aid $i$ at node $n$.

$\iota_{\text {in }}^{-} \quad$ Unit penalty cost for the shortage of emergency aid $i$ at node $n$.

$o_{i n} \quad$ Unit procurement cost of emergency aid i at node $n$.

$\rho_{i n} \quad$ Unit prepositioning cost for emergency aid $i$ at node $n$ (procurement plus opportunity cost).

\section{Stochastic input data}

$b_{i n t}^{\xi} \quad$ Incoming supplies of emergency aid $i$ at node $n$ in period $t$ in scenario $\xi$.

$d_{\text {int }}^{\xi} \quad$ Demand for emergency aid $i$ at node $n$ in period $t$ in scenario $\xi$.

$f_{t}^{\xi} \quad$ Financial budget available in period $t$ in scenario $\xi$ (donations, federal funding, etc.).

$q_{r n m t}^{\xi}=1$ if a vehicles of type $r$ can cross arc $n \rightarrow m$ in period $t$ in scenario $\xi$ $=0$ if not.

$s_{i n}^{\xi} \quad$ Fraction of the prepositioned emergency aid $i$ at node $n$ that remains usable in the first period in scenario $\xi$.

\section{First-stage decisions}

$P_{i n}^{0} \quad$ Amount of emergency aid $i$ prepositioned at node $n$ at the start of the first period.

$Y_{r}^{C} \quad$ Number of vehicles of type $r$ contracted in the first-stage to transport emergency aid over the periods.

\section{Second-stage decisions}

$I_{\text {int }}^{\xi+} \quad$ Available inventory of emergency aid $i$ at node $n$ in period $t$ in scenario $\xi$.

$I_{\text {int }}^{\xi-} \quad$ Shortage (unmet demand) of emergency aid $i$ at node $n$ in period $t$ in scenario $\xi$.

$\mathcal{O}_{\text {int }}^{\xi} \quad$ Amount of procured emergency aid $i$ at node $n$ in period $t$ in scenario $\xi$.

$V_{r n m t}^{\xi} \quad$ Number of vehicles of type $r$ used on $\operatorname{arc} n \rightarrow m$ in period $t$ in scenario $\xi$.

$X_{\text {irnmt }}^{\xi} \quad$ Flow of emergency aid $i$ shipped by vehicles of type $r$ on $\operatorname{arc} n \rightarrow m$ in period $t$ in scenario $\xi$.

$Z_{t}^{\xi} \quad$ Unused budget at the end of period $t$ in scenario $\xi$.

The risk-neutral two-stage stochastic network model for emergency logistics planning is formulated 
as follows:

(F1: RiskNeutral)

$$
\begin{array}{ll}
\text { Min } & \sum_{i \in \mathscr{I}} \sum_{n \in \mathscr{N}^{w}} \rho_{i n} \cdot P_{i n}^{0}+\sum_{r \in \mathscr{R}} \beta_{r} \cdot Y_{r}^{C}+\sum_{i \in \mathscr{I}} \sum_{n \in \mathscr{N}} \sum_{t \in \mathscr{T}} \sum_{\xi \in \Xi} \pi^{\xi} \cdot\left(\iota_{i n}^{+} \cdot I_{i n t}^{\xi+}+\iota_{i n}^{-} \cdot I_{i n t}^{\xi-}\right) \\
\text { s.t.: } & \sum_{n \in \mathscr{N}^{w}} P_{i n}^{0} \leq p_{i}^{\max }, \quad i \in \mathscr{I} \\
& Y_{r}^{C} \leq c_{r}^{\max }, \quad r \in \mathscr{R}
\end{array}
$$

$\sum_{r \in \mathscr{R}} \sum_{m \in \mathscr{N}}\left[X_{i r n m t}^{\xi}-X_{i r m n\left(t-\ell_{r m n t}\right)}^{\xi}\right]=\left\{\begin{array}{c}I_{i n(t-1)}^{\xi+}-I_{i n(t-1)}^{\xi-}+I_{i n t}^{\xi-}-I_{i n t}^{\xi+}+\mathcal{O}_{i n t}^{\xi}+b_{i n t}^{\xi}-d_{i n t}^{\xi}, \\ i \in \mathscr{I}, n \in \mathscr{N}^{r c}, t \in \mathscr{T}, \xi \in \Xi \\ I_{i n(t-1)}^{\xi+}-I_{i n t}^{\xi+}+s_{i n}^{\xi} \cdot P_{i n}^{0}+b_{i n t}^{\xi}, \\ i \in \mathscr{I}, n \in \mathscr{N}^{w}, t=1, \xi \in \Xi \\ I_{i n(t-1)}^{\xi+}-I_{i n t}^{\xi+}+b_{i n t}^{\xi}, \\ i \in \mathscr{I}, n \in \mathscr{N}^{w}, t \in \mathscr{T}-\{1\}, \xi \in \Xi\end{array}\right.$

$$
\begin{aligned}
& \sum_{t \in \mathscr{T}} \mathcal{O}_{i n t}^{\xi} \leq o_{i n}^{\max }, i \in \mathscr{I}, n \in \mathscr{N}^{r c}, \xi \in \Xi \\
& V_{r n m t}^{\xi} \geq \sum_{i \in \mathscr{I}} \frac{c_{i}^{w}}{g_{r}^{w}} \cdot X_{i r n m t}^{\xi}, r \in \mathscr{R},(n, m) \in \mathscr{N}, t \in \mathscr{T}, \xi \in \Xi
\end{aligned}
$$

$$
V_{r n m t}^{\xi} \geq \sum_{i \in \mathscr{I}} \frac{c_{i}^{v}}{g_{r}^{v}} \cdot X_{i r n m t}^{\xi}, r \in \mathscr{R},(n, m) \in \mathscr{N}, t \in \mathscr{T}, \xi \in \Xi
$$

$$
\sum_{(n, m) \in \mathscr{N}} \sum_{t \in \mathscr{T}} V_{r n m t}^{\xi} \leq Y_{r}^{C}, r \in \mathscr{R}, r \in \mathscr{R}, \xi \in \Xi
$$

$$
V_{r n m t}^{\xi} \leq q_{r n m t}^{\xi} \cdot Y_{r}^{C}, r \in \mathscr{R},(n, m) \in \mathscr{N}, t \in \mathscr{T}, \xi \in \Xi
$$

$$
\begin{aligned}
& \sum_{r \in \mathscr{R}} \sum_{(n, m) \in \mathscr{N}} \alpha_{r n m} \cdot V_{r n m t}^{\xi}+\sum_{i \in \mathscr{I}} \sum_{n \in \mathscr{N}^{r c}} o_{i n} \cdot \mathcal{O}_{i n t}^{\xi}=f_{t}^{\xi}+Z_{(t-1)}^{\xi}-Z_{t}^{\xi}, t \in \mathscr{T}, \xi \in \Xi \\
& Y_{r}^{C} \geq 0 \text { and integer, } r \in \mathscr{R} \\
& V_{\text {rnmt }}^{\xi} \geq 0 \text { and integer }, r \in \mathscr{R},(n, m) \in \mathscr{N}, t \in \mathscr{T}, \xi \in \Xi \\
& P_{i n \mid n \in \mathscr{N}^{w}}^{0}, X_{\text {irnmt }}^{\xi}, I_{\text {int }}^{\xi+}, I_{\text {int }}^{\xi-}, \mathcal{O}_{i n t \mid n \in \mathscr{N}^{r c}}^{\xi}, Z_{t}^{\xi} \geq 0, i \in \mathscr{I}, r \in \mathscr{R},(n, m) \in \mathscr{N}, t \in \mathscr{T}, \xi \in \Xi .
\end{aligned}
$$

The objective function (1) minimizes a weighted sum of the first-stage cost of prepositioning stock, hiring vehicles, and the expected second-stage cost of inventory and unmet demand. Constraints (2) limit the amount of each emergency aid that can be prepositioned across the warehouse nodes and can be easily modified if a specific node has tight capacity. Constraints (3) limit the total quantity of each type of vehicle that can be contracted to transport emergency aid over the humanitarian operations. 
The flow constraints (4) balance inventory, unmet demand, procurement and the prepositioning of emergency aid with demand and incoming supplies over consecutive time periods at each node in the network. The first equality in (4) represents the flow through nodes with demand (relief centers), where there could be supply and procurement. The second equality guarantees the flow of the available quantity of emergency aid (supplies and/or prepositioned goods) through nodes without demand (warehouses) in the first-period. In this case, prepositioning is possible, but shortages are not allowed. The last equality is similar, but it refers to the second time period onwards, in which case prepositioning is not a valid policy. Without loss of generality, initial inventory and unmet demand (in $t=0$ ) are considered to be zero. Note that constraints (4) combined with the minimization of inventory and unmet demand in the objective function (1) means that material convergence clogging is lessened by not pushing unwanted donated supplies into the supply chain.

Constraints (5) limit the amount of emergency aid that can be procured across the relief center nodes. Constraints (6) and (7) determine the type and number of vehicles required to transport emergency aid through the network. A number of different vehicles, such as trucks, boats and helicopters, are likely to be used to reach precarious areas, such as flooded and collapsed regions. Both weight and volume loading are considered to determine the most suitable vehicles, as a particular emergency aid can be light-weight but bulky, or vice-versa. Constraints (8) guarantee that the total number of vehicles used to transport emergency aid after disaster strikes is not greater than the fleet of vehicles contracted in the first-stage. Constraints (9) link the number of vehicles required in each route, period and scenario to the available arcs. For example, depending on the disaster, $q_{r n m t}^{\xi}$ might be zero for road transportation, but not necessarily for air transportation. If $q_{r n m t}^{\xi}=1$, then the number of vehicles of type $r$ used in the second-stage is limited by $Y_{r}^{C}$, which is a sufficiently large number in this case. Constraints (10) ensure that the total cost of using vehicles to ship emergency aid plus the procurement cost stays within the financial budget. Constraints (11), (12) and (13) specify the domains of the decision variables.

\section{Model Extensions via Risk Management}

Although the scope of this paper does not include a comprehensive analysis of possible extensions of the proposed stochastic model, we also analyse some models from the risk management viewpoint. The research question that motivates this discussion is: "Can risk-averse models improve humanitarian operations optimized by the risk-neutral model, by, for example, reducing a key variability measure or mitigating the risk of non-wanted scenarios?".

To answer, we investigated the classical minimax-regret approach (MinMax) and two state-of-art risk risk measures: semideviation (SD) and conditional value-at-risk (CVaR). The reasons behind the choice of those risk measures are as follows. Minimax is well-known and used in the literature, and various authors claim that it can provide "robust" solutions from a worst-case scenario perspective (Kouvelis and Yu, 1997; Shapiro and Ahmed, 2004; Riis and Andersen, 2005). It means that optimal solutions present relatively good stability regarding perturbation of the underline distribution. This strategy can be particularly useful when the probability of the different scenarios is difficult to estimate accurately, or simply when we cannot assess the probability distribution due to a lack of historical data, for example. In this case, though, scenarios can be estimated based on statistical information on the random data. 
The motivation for adopting semideviation and CVaR as risk measures are their coherency, as well as their consistency with stochastic dominance principles - thus allowing us to search for stochastically non-dominated solutions (Ogryczak and Ruszczyński, 2001). Moreover, the corresponding optimization problems are computationally tractable (due to the convexity of the risk measures), which is an appealing feature when dealing with mixed-integer problems.

The minimax-with-regret aims to obtain the best worst-case deviation from optimality among all feasible decisions over the entire set of scenarios (Kouvelis and $\mathrm{Yu}, 1997$ ). The regret is simply measured as the difference between the value of the recourse cost and the value of the corresponding scenario $W^{\star}(\xi)$. The resulting Minmax problem is as follows:

(F2: Minmax)

Min $\Phi$

$$
\begin{aligned}
& \text { s.t. } \text { Constraints }(2)-(13) \\
& \Phi \geq \sum_{i \in \mathscr{I}} \sum_{n \in \mathscr{N} w} \rho \cdot P_{i n}^{0}+\sum_{r \in \mathscr{R}} \beta_{r} \cdot Y_{r}^{C}+ \\
& \quad+\sum_{i \in \mathscr{I}} \sum_{n \in \mathscr{N}} \sum_{t \in \mathscr{T}}\left[\iota_{i n}^{+} \cdot I_{\text {int }}^{\xi+}+\iota_{\text {in }}^{-} \cdot I_{\text {int }}^{\xi-}\right]-W^{\star}(\xi), \xi \in \Xi \\
& \Phi \geq 0,
\end{aligned}
$$

where $W^{\star}(\xi)$ is the optimal value for the single scenario wait-and-see problem.

The CVaR risk measure accounts for the expected cost of exceeding VaR at confidence level $u$. VaR is the so-called value-at-risk, i.e., the loss that is expected to be exceeded with a small probability $(1-u)$ during a certain time period. CVaR is defined as follows:

$$
\operatorname{CVaR}[Q(\mathscr{Y}, \xi)]=\inf _{\operatorname{VaR} \in \mathbb{R}}\left\{\eta+\frac{1}{1-u} \mathbb{E}[Q(\mathscr{Y}, \xi)-\operatorname{VaR}]_{+}\right\}
$$

where $\mathbb{E}(\cdot)$ is the expected value a random variable, $[Q(\mathscr{Y}, \xi)-\mathrm{VaR}]_{+}=\max \{0, Q(\mathscr{Y}, \xi)-\mathrm{VaR}\}, u \in$ $(0,1)$, and $Q(\mathscr{Y}, \xi)$ is the random recourse that depends upon the first-stage decisions $\mathscr{Y}$. By assuming a finite support $\Xi$ with probabilities $\pi^{1}, \pi^{2}, \cdots, \pi^{\mid} \Xi \mid$ associated to the instances $\xi^{1}, \xi^{2}, \cdots, \xi^{|\Xi|}$, then problem CVaR becomes:

\section{(F3: $\quad$ CVaR)}

Min $\sum_{i \in \mathscr{I}} \sum_{n \in \mathscr{N} w} \rho_{i n} \cdot P_{i n}+\sum_{r \in \mathscr{R}} \beta_{r} \cdot Y_{r}^{C}+(1-\phi) \cdot \sum_{i \in \mathscr{I}} \sum_{n \in \mathscr{N}} \sum_{t \in \mathscr{T}} \sum_{\xi \in \Xi} \pi^{\xi} \cdot\left[\iota_{i n}^{+} \cdot I_{i n t}^{\xi+}+\iota_{i n}^{-} \cdot I_{i n t}^{\xi-}\right]+$ $+\phi \cdot\left(\operatorname{VaR}+\frac{1}{1-u} \cdot \sum_{\xi \in \Xi} \pi^{\xi} \cdot \Theta^{\xi}\right)$

s.t. Constraints $(2)-(13)$

$$
\begin{aligned}
& \Theta^{\xi} \geq \sum_{i \in \mathscr{I}} \sum_{n \in \mathscr{N}} \sum_{t \in \mathscr{T}}\left[\iota_{i n}^{+} \cdot I_{i n t}^{\xi+}+\iota_{i n}^{-} \cdot I_{i n t}^{\xi-}\right]-\mathrm{VaR}, \xi \in \Xi \\
& \Theta^{\xi} \geq 0, \xi \in \Xi, \text { VaR free, }
\end{aligned}
$$

where $\phi \in[0,1]$ is a weight that serves to trade off expected value and risk and $u$ is the confidence level. Variable $\Theta^{\xi}$ is 0 if scenario $\xi$ has a total cost lower than the $\mathrm{VaR}$, otherwise $\Theta^{\xi}$ is the difference between $\mathrm{VaR}$ and the corresponding cost for all $\xi$. Both $\phi$ and $u$ reflect risk or robust preferences. As $u$ increases, 
fewer worst-case scenarios are taken into account. Consequently, recourse costs are expected to be more stable across the different scenarios.

The semideviation measure of order $p$ is defined as follows:

$$
\mathbb{D}_{p}=\mathbb{E}\left[(Q(\mathscr{Y}, \xi)-\mathbb{E} Q(\mathscr{Y}, \xi))^{p}\right]^{1 / p}, \forall p \geq 1
$$

For $p=1$, the semideviation of order 1 or the mean-semideviation measure (Krokhmal et al, 2011) can be stated as follows:

$$
\mathbb{D}_{p}=\mathbb{E} \max [Q(\mathscr{Y}, \xi)-\mathbb{E} Q(\mathscr{Y}, \xi) ; 0]=\mathbb{E} \max [Q(\mathscr{Y}, \xi) ; \mathbb{E} Q(\mathscr{Y}, \xi)] .
$$

There are different ways to linearise expression (16). We use the straightforward linearisation proposed by Markert and Schultz (2005) who use an auxiliary variable, e.g. $\Delta^{\xi}$, to account for the maximum value in equation (16). Under a finite number of scenarios, model SD is finally written as

$$
\begin{array}{ll}
\text { (F4: } & \text { SD }) \\
\text { Min } & \sum_{i \in \mathscr{I}} \sum_{n \in \mathscr{N}} \rho_{i n} \cdot P_{i n}+\sum_{r \in \mathscr{R}} \beta_{r} \cdot Y_{r}^{C}+(1-\phi) \cdot \sum_{i \in \mathscr{I}} \sum_{n \in \mathscr{N}} \sum_{t \in \mathscr{T}} \sum_{\xi \in \Xi} \pi^{\xi} \cdot\left[\iota_{\text {in }}^{+} \cdot I_{\text {int }}^{\xi+}+\iota_{\text {in }}^{-} \cdot I_{\text {int }}^{\xi-}\right]+ \\
& +\phi \cdot \sum_{\xi \in \Xi} \pi^{\xi} \cdot \Delta^{\xi} \\
\text { s.t. } \quad & \text { Constraints }(2)-(13) \\
& \sum_{i \in \mathscr{I}} \sum_{n \in \mathscr{N}} \sum_{t \in \mathscr{T}}\left[\iota_{i n}^{+} \cdot I_{\text {int }}^{\xi+}+\iota_{\text {in }}^{-} \cdot I_{\text {int }}^{\xi-}\right]-\Delta^{\xi} \leq 0, \xi \in \Xi \\
& \sum_{i \in \mathscr{I}} \sum_{n \in \mathscr{N}} \sum_{t \in \mathscr{T}} \sum_{\xi^{\prime} \in \Xi} \pi^{\xi^{\prime}} \cdot\left[\iota_{\text {in }}^{+} \cdot I_{\text {int }}^{\xi^{\prime}+}+\iota_{\text {in }}^{-} \cdot I_{\text {int }}^{\xi^{\prime}-}\right]-\Delta^{\xi} \leq 0, \xi \in \Xi \\
& \Delta^{\xi} \geq 0, \xi \in \Xi
\end{array}
$$

where the two new constraints in (17) together with the minimization of the risk term $\sum_{\xi \in \Xi} \pi^{\xi} \cdot \Delta^{\xi}$ define the semideviation risk measure represented by the second-stage decision variable $\Delta^{\xi}$. Note that both CVaR and SD are mean-risk models with an objective function that is able to produce risk-neutral solutions for $\phi=0$, solutions that are totally risk-averse for $\phi=1$, or solutions that consider simultaneously both features for $\phi \in(0,1)$.

Table 2 summarizes the structure of the proposed risk-averse models in terms of necessary inputs and outputs.

[Insert Table 2 here.]

\section{Solution Procedure}

Solving the proposed (risk neutral and risk-averse) two-stage stochastic mixed-integer programming models turns out to be computationally challenging. This is especially due to the number of integer decision variables, $|\mathscr{R}|+|\mathscr{R}||\mathscr{N}|^{2}|\mathscr{T}||\Xi|$, which can be large, even for small instances. Using the heuristic developed just below, this problem dimensionality is manageable for the real Brazilian network instance 
used in the computational tests in Section 6. However, it may well not be for some of the more complex and larger networks encountered in practice. Testing on larger instances will be required in future research.

In order to provide good-quality solutions within a reasonable amount of time, we propose a simple but effective two-phase heuristic. In the first phase, a simplified version of the mathematical model is solved in order to obtain transportation decisions $\left(X_{\text {irnmt }}^{\xi}\right)$. In the second phase, the original formulation is solved with the information gathered from the first phase and the remaining decision variables are determined. The overall approach is illustrated in Figure 2.

[Insert Figure 2 here]

The simplified model SMIP (17) links the first-stage decisions $Y_{r}^{C}$ directly with the second-stage flow variables $X_{\text {irnmt }}^{\xi}$ via the parameters $\frac{c_{i}^{w}}{g_{r}^{w}}$ and/or $\frac{c_{i}^{v}}{g_{r}^{v}}$, without needing the integer decision variables $V_{r n m t}^{\xi}$.

$$
(\mathrm{SMIP})\left\{\begin{array}{l}
\text { Min expression }(1) \\
\text { Constraints }(2),(3),(4),(5),(11),(13) \\
q_{r n m t}^{\xi} \cdot Y_{r}^{C} \geq \sum_{i \in \mathscr{I}} \frac{c_{i}^{w}}{g_{r}^{w}} \cdot X_{i r n m t}^{\xi}, r \in \mathscr{R},(n, m) \in \mathscr{N}, t \in \mathscr{T}, \xi \in \Xi \\
q_{r n m t}^{\xi} \cdot Y_{r}^{C} \geq \sum_{i \in \mathscr{I}} \frac{c_{i}^{v}}{g_{r}^{v}} \cdot X_{i r n m t}^{\xi}, r \in \mathscr{R},(n, m) \in \mathscr{N}, t \in \mathscr{T}, \xi \in \Xi \\
Y_{r}^{C} \geq \sum_{i \in \mathscr{I}} \sum_{(n, m) \in \mathscr{N}} \sum_{t \in \mathscr{T}} \frac{c_{i}^{w}}{g_{r}^{w}} \cdot X_{i r n m t}^{\xi}, r \in \mathscr{R}, \xi \in \Xi \\
Y_{r}^{C} \geq \sum_{i \in \mathscr{I}} \sum_{(n, m) \in \mathscr{N}} \sum_{t \in \mathscr{T}} \frac{c_{i}^{v}}{g_{r}^{v}} \cdot X_{i r n m t}^{\xi}, r \in \mathscr{R}, \xi \in \Xi \\
\sum_{i \in \mathscr{I}} \sum_{r \in \mathscr{R}} \sum_{(n, m) \in \mathscr{N}} \alpha_{r n m} \cdot \max \left\{\sum_{i \in \mathscr{I}} \frac{c_{i}^{w}}{g_{r}^{w}} \cdot X_{i r n m t}^{\xi}, \sum_{i \in \mathscr{I}} \frac{c_{i}^{v}}{g_{r}^{v}} \cdot X_{i r n m t}^{\xi}\right\} \\
\quad+\sum_{i \in \mathscr{I}} \sum_{n \in \mathscr{N}^{r c}} o_{i n} \cdot \mathcal{O}_{i n t}^{\xi}=f_{t}^{\xi}+Z_{(t-1)}^{\xi}-Z_{t}^{\xi}, t \in \mathscr{T}, \xi \in \Xi .
\end{array}\right.
$$

The non-linear budget constraint in (17) is then approximated by the linearisation (18) in which both the weight and volume of the emergency aid are taken into account to avoid infeasible solutions in the second phase. Subsection 6.6 shows that the differences between the exact and approximated solutions are negligible from the practical point of view, even though the number of vehicles in the first-stage is overestimated due to the linearisation:

$$
\sum_{r \in \mathscr{R}} \sum_{(n, m) \in \mathscr{N}} \alpha_{r n m} \cdot \sum_{i \in \mathscr{I}}\left(\frac{c_{i}^{w}}{g_{r}^{w}}+\frac{c_{i}^{v}}{g_{r}^{v}}\right) \cdot X_{i r n m t}^{\xi}+\sum_{i \in \mathscr{I}} \sum_{n \in \mathscr{N}^{r c}} o_{i n} \cdot \mathcal{O}_{i n t}^{\xi}=f_{t}^{\xi}+Z_{(t-1)}^{\xi}-Z_{t}^{\xi}
$$

for all $t \in \mathscr{T}$ and $\xi \in \Xi$.

In the first phase, we still have a two-stage stochastic mixed-integer programming model, but with only $|\mathscr{R}|$ integer decisions. In practice, as the number of different types of vehicles (modes of transportation) is relatively small, we expect to obtain (sub)optimal solutions rapidly. Note that the simplified model only serves to provide the flow decisions for the original problem. Given the flow variables, $V_{r n m t}^{\xi}$ is trivially determined, as well as the overall solution of the original problem. We also tested the two-phase 
procedure by varying the fixation criteria, but found that the most encouraging results are obtained by fixing the flow variables only. In the computational tests, we show that this procedure is indeed more efficient than solving the exact models directly via CPLEX. Note that the two-phase procedure is suitable for solving the risk-averse models as well.

\section{Computational Tests}

The aim of this section is to analyse the performance of the proposed mathematical models and provide practical insights that can help decision-makers in the humanitarian logistics field. We also show the efficiency of the solution procedure in finding good-quality solutions within a reasonable amount of time. The mathematical model was tested and analysed using data from the flooding and landslide disaster in January 2011 in the Serrana region of Rio de Janeiro state, Brazil.

All the mathematical models were coded in the GAMS 24.0.2 modelling language and solved with the CPLEX 12.5 solver. Preliminary tuning experiments with GAMS/CPLEX led us to adopt the following nondefault options: do not solve the fixed problem ("solvefinal =0"); use compressed node files on disk ("nodefileind $=3$ "); enable an opportunistic parallel search mode ("parallelmode $=-1$ "); and use all but one core ("threads $=-1$ "). The stopping criteria were either elapsed times exceeding 14,400 seconds or optimality gaps relative to the best lower bound smaller than 0.01. The experiments were performed on an Intel Core i7 computer with 16 GB RAM and the Windows 7 operating system.

\subsection{Brief description of the case-study}

According to the state Geological Service (DRM, 2011), the disaster in January 2011 in the Serrana region of Rio de Janeiro state, Brazil, occurred due to three main factors: $(i)$ predisposing factors, such as geology, geomorphology and the climate of the region; ( $i i)$ active factors, such as land use and occupation, previous rainfall, pre-storm rainwater and fluvial erosion; and ( $i i i)$ the igniting factor, i.e., torrential rain that lasted 4 hours. The Brazilian Yearbook of Natural Disasters (Brasil, 2012) pointed out that the megadisaster caused hundreds of deaths and thousands of homeless and displaced people. It was the largest disaster ever recorded in Brazil in number of fatalities, causing Serrana to be the Brazilian region with the highest number of deaths caused by a natural disaster (40\% of the national total between 1988 and 2012).

According to the categorization scheme proposed by Apte (2009), the megadisaster is a type III disaster, being a sudden onset from the temporal coverage viewpoint, and a localized onset from the spatial coverage standpoint. In fact, many floods and flash floods are sudden events. Despite the fact that the megadisaster reached several cities, it is still considered a geographically contained event, mainly in comparison to the 2004 Indian Ocean Tsunami that affected 14 dispersed countries. Figure 4 depicts the complete categorisation and provides some examples of disaster events for each category.

Basically, the idea behind this classification is to understand and analyse the level of difficulty that each type of disaster imposes for carrying out humanitarian operations in terms of geographical/spatial and temporal coverage. Higher uncertainty levels are also reflected in more difficult humanitarian operations. For example, prepositioning strategies can be more challenging in sudden onset and localized disasters than in sudden onset and dispersed (more uncertain) disasters as, in the second case, extra 
coordination mechanisms are needed among the players in the humanitarian supply chain in order to provide fast relief, even with decentralized stockpiles of emergency aid (Apte, 2009). Note too that a dispersed and slow onset disaster (type II), such as the 2015-16 Zica virus epidemic in Brazil and parts of Latin America, presents different levels of difficulty regarding humanitarian operations, but given that it is not a sudden event, humanitarian logisticians can benefit from time to prepare for relief operations.

[Insert Figure 4 here.]

\subsection{Data of the base case}

The base case was designed to be as realistic as possible through interviews with governmental organisations, and by extracting information from a variety of technical reports, websites, and existing literature (Rawls and Turnquist, 2010; Lin et al, 2012; Peixoto Filho, 2013; dos Santos, 2013; Brasil, [n.d.]). Due to the limited availability of information on Brazilian disasters, some parameters were estimated from previous papers, so the example should be treated as illustrative. For the sake of brevity, we present the main data here, but the detailed description of the remaining parameters are presented in the online supplementary content (Appendix A.1; see Tables A, B, C, D, and E).

The requirement is to provide humanitarian aid to fulfill daily demand over a horizon of 7 periods of 1 day each by supplying emergency emergency aid across a network structure composed of 9 relief centers (demand nodes) and 4 depots (non-demand nodes). Motivated by real-life cases, the examples assume that prepositioning and incoming supplies occur only at depot nodes and procurement only at demand nodes from the first time period onwards. However, it is possible to transport emergency aid across any arc $n \rightarrow m$ ( $n$ and $m$ being relief centers and/or depots) as long as it is available. Initially, all arcs are assumed to be available for any type of vehicle $\left(q_{r n m t}^{\xi}=1\right)$ and that both the capacities $c_{r n m}^{\max }$ and $c_{r}^{\max }$ are sufficiently large.

The following nine affected areas (demand nodes) were considered: Teresópolis (TRS), Petrópolis (PTP), Nova Friburgo (NFB), São José do Vale do Rio Preto (SVRP), Bom Jardim (BJD), Sumidouro (SMD), Areal-Sapucaia-Três Rios (AST), Santa Maria Madalena (SMM) and São Sebastião do Alto (SSA). There are four depots (non-demand nodes) located in Teresópolis (TRS-D), Petrópolis (PTP-D), Nova Friburgo (NFR-D) and Rio de Janeiro city (RJ-D). Figure 3 shows the district map of Rio de Janeiro state where Serrana is the inland central region.

[Insert Figure 3 here.]

We consider six emergency emergency aid items that are commonly distributed among disaster victims: food, water, hygiene, cleaning products, medical products, and mattress. A kit of food represents a basic food basket covering the nutritional requirements of a five-person family. Each unit of water represents a container with 20 litres. A kit of personal hygiene products serves only one person. A unit of cleaning products (or one kit) serves a five-person family. On the other hand, a kit of medical products serves 90 people. A mattress serves one person. A summary of these requirements, the characteristics of the emergency aid (weight $c_{i}^{w}$, volume $c_{i}^{v}$, the prepositioning cost per emergency aid $\rho_{i n}$, and the maximum amount of each emergency aid that can be prepositioned $\left(p_{i}^{\max }\right)$ is shown in Table 3 . Prepositioning costs correspond to the procurement price of emergency aid before a disaster strikes; the penalty for 
unmet demand of each emergency aid item $\left(\iota_{i n}^{-}\right)$is 100 times greater than the procurement price, and holding costs $\left(\iota_{i n}^{+}\right)$are $25 \%$ of the procurement price. Procurement costs after a disaster strikes $\left(o_{i n}\right)$ are 5 times those before the disaster. The procurement capacity for each emergency aid and node $\left(o_{i n}^{\max }\right)$ is limited to $20 \%$ of the corresponding prepositioning capacity.

[Insert Table 3 here]

We consider three types of vehicle: trucks, boats and helicopters, whose capacities and costs are summarized in Table E in Appendix A.1. Shipping costs were evaluated as follows:

$$
\alpha_{r n m}=\frac{\text { fuel }(r)}{\operatorname{consumption}(r)} \cdot \operatorname{dist}(n, m),
$$

where $\operatorname{dist}(n, m)$ is the distance (in $\mathrm{km}$ ) between two nodes. In the absence of the exact address of the relief center or depot, the distance was approximated via the centroid of each area.

Finally, the total monetary donation $\left(f_{t}\right)$ was estimated to be 25 million Brazilian Reais (BRL) in the first period only. Lead times were assumed to be 1 day only for the first time period and for truck vehicles. The remaining lead times were considered to be zero.

\subsection{Scenario Generation}

To draw up plausible scenarios, historical data from previous disasters was investigated and classified according to the scaling system proposed by Eshghi and Larson (2008), who categorized any disaster in one of six magnitude classes: emergency situation, crisis situation, minor disaster, moderate disaster, major disaster and catastrophe. Through extensive data sourced from the Emergency Events Database EM-DAT (Centre for Research on the Epidemiology of Disasters, 2014) of the last 105 years of disasters around the world, the distribution of disasters was analysed for different values of fatal and affected victims. The output of this analysis consists of (a) six ranges or intervals for the number of fatal victims and the corresponding proportion of disasters that fall within each interval; and (b) six ranges or intervals for the number of affected victims and the corresponding proportion of disasters that fall within each interval. For example, it was concluded that $78.1 \%$ of all disasters cause the number of fatal victims to be in the interval $(10 ; 100]$. We now present the main data for the scenario generation procedure. Additional data are presented in the online supplementary content (Appendix A.2; see Tables F, G, H, and I).

The magnitude class of a particular disaster is calculated from the number of fatal $\left(\mu^{+}\right)$and affected victims $(\mu)$ using a mathematical expression to represent the impact of the disaster, as follows:

$$
\psi=\max \left\{\frac{\mu^{+}-L_{\mu^{+}}}{U_{\mu^{+}}}+\log L_{\mu^{+}}, \frac{\mu-L_{\mu}}{U_{\mu}}+\log L_{\mu}-1\right\},
$$

where $L_{\mu^{+}}\left(L_{\mu}\right)$ and $U_{\mu^{+}}\left(U_{\mu}\right)$ are the lower $(\mathrm{L})$ and upper $(\mathrm{U})$ bounds of the intervals that contain the exact number of fatal (affected) victims. We then analysed the value of $\psi$ to further classify the type of the disaster; see Table F in Appendix A.2 for the detailed intervals proposed for the number of fatal and affected victims, as well as the value of the magnitude $\psi$ of each disaster and its corresponding classification. 
Based on this methodology, we determined the magnitude $\psi$ and classified the disasters in the state of Rio de Janeiro from 1966 to 2013, as depicted in Table G in Appendix A.2. For example, consider the last recorded disaster that caused 67 fatal victims and 2,300 affected people for which $\mu^{+}=67$ and $\mu=2,300$. The number of victims lies in the interval $(10 ; 100]$ and the number of affected people in $(1,000 ; 10,000]$, so that $L_{\mu^{+}}=10, U_{\mu^{+}}=100, L_{\mu}=1,000, U_{\mu}=10,000$. Thus the magnitude $\psi$ is evaluated as follows:

$$
\psi=\max \left\{\frac{67-10}{100}+\log 10 ; \frac{2,300-1,000}{10,000}+\log 1,000-1\right\}=\max \{1.57 ; 2.13\}=2.13,
$$

which lies on the interval $[2,3)$ and corresponds to an emergency situation (as classified in Table G).

We used the frequency Table $\mathrm{H}$ in Appendix A.2 to estimate the probability of occurrence of all types of disasters in the state of Rio de Janeiro. The transition probabilities among different types of disasters are shown in Table I in Appendix A.2. Note that, although there are only 21 recorded events, they cover a period of 47 years of analysis. For this reason, we assume that the relative frequency of each disaster event can be used as a reasonable approximation for the underlying probability distribution.

The number of affected people (column 3 in Table G) corresponds not only to the displaced and homeless, but also to anyone who was directly or indirectly affected. Thus only some of these victims need emergency supplies. The actual number of victims requiring aid is estimated via the percentage of displaced, homeless and fatal victims from the overall number of affected people in the disaster of 2011, being $10.96 \%$ (33,370 victims from 304,562 total affected). Thus we use this percentage to estimate the amount of needed emergency aid for all scenarios. The daily number of victims was generated using a random-based generator that we devised for this paper. Basically, the random generator assumes that large amounts of demand occur abruptly over short lead times (Sarkis et al, 2010), contrasting with periods of low demand.

The other stochastic parameters were taken into account as follows: a first scenario is constructed with the same magnitude as the demand; a second scenario with magnitude one demand level lower; and a third scenario with magnitude one level higher. For example, a moderate demand scenario splits into 3 supply-donation pair scenarios corresponding to a minor disaster (one level below), a moderate disaster (same level), and a major disaster (one level above). This $(-1,0,+1)$ split assumes that the amount of collected supplies and donations will not follow exactly the magnitude of the disaster, but could be somewhat lower or higher. It also assumes that the route damage is partially proportional to the number of victims in the sense that worse disasters provoke more damage, but major disasters could cause slightly less damage (as in minor disasters), or slightly worse damage (as in catastrophic situations). The conditional probabilities among states $(-1,0,+1)$ are assumed to be those in Table J of the supplementary material. Thus, for example, scenario 23 has

$\pi[$ demand is moderate $]=0.1429$

$\pi[$ supply-donation is minor-minor $\mid$ demand is moderate $]=0.25$

$\pi$ [damage is minor $\mid$ demand is moderate] $=0.25$ 
so that the corresponding probability of scenario 1 [moderate, minor-minor, minor] $=0.1429 \times 0.25 \times$ $0.25=0.0089$.

The resulting set of scenarios is the combination of the four stochastic parameters, totalling 40 scenarios, as depicted in Figure 5. Statistical independence among the parameters is assumed so that the probability of each scenario is determined by the product of the probabilities of each stochastic parameter. It is worth noting that our proposed scenarios are not time-dependent. Thus we simply independently generate a number of instances over the full horizon and combined them in the proposed scenario-tree.

[Insert Figure 5 here]

Note that the categorisation based on temporal and spatial coverage (Apte, 2009) previously discussed could be used in conjunction with this current scale system to generate alternative scenarios in an attempt to analyse, for example, the effectiveness of both preparation and response operations when localized and dispersed disasters occur at the same time in Brazilian territory. Testing via alternative scenario use is left for future research.

\subsection{Presentation of the Test Cases}

Based on the nominal data (base case), we construct 16 additional cases, totalling 17 instances. The motivation in proposing extra instances is to obtain insights into the performance of the model over a spectrum of practical situations commonly observed in humanitarian contexts. These cases were designed with the same parameters as the base case (A0), but by considering different assumptions, as follows. (A1) without prepositioning; (A2) prepositioning in all nodes is allowed; (A3) 25\% of stockpiling is lost; (A4) procurement is not allowed; (A5) doubled supply; (A6) doubled donations; (A7) doubled supply and donations; (A8) without damaged arcs; (A9) with more damaged arcs; (A10) without trucks; (A11) without helicopters; (A12) without trucks and boats; (A13) truck capacity is 5 times larger; (A14) without lead time; (A15) objective function prioritises medicine kits; (A16) scenarios are equiprobable. These 16 variants from the base case overlap to some extent and are not exhaustive, but do provide an interesting range of representative cases against which to test the model.

\subsection{Numerical Results and Managerial Insights}

This section presents the numerical results and discusses the managerial insights. Additional material to support our insights is presented in the online supplementary content (Appendix A.3; see Tables J, K, L, M, N, O, and P, and Figure A).

In stochastic programming, it is common to (i) evaluate the impact of randomness in the problem, and (ii) analyse if simpler approaches (e.g., replacing all random variables by their expected values and solving the resulting problem) are sufficient to take uncertainty into account. These two issues can be addressed by analysing the Expected Value of Perfect Information (EVPI) and the Value of Stochastic Programming (VSS) (Birge and Louveaux, 1997). EVPI compares stochastic with wait-and-see approaches and provides an idea of how a solution would be under perfect information. It is computed as the difference between the objective function (RP) of the stochastic programming model (the recourse problem) and the expected wait-and-see solution (WS), i.e., EVPI $=\mathrm{RP}-\mathrm{WS}$, where $\mathrm{WS}=\sum_{\xi \in \Xi} \pi^{\xi} W^{\star}(\xi)$ and $W^{\star}(\xi)$ is the singlescenario deterministic problem associated with scenario $\xi$. VSS compares stochastic with expected value 

approaches (whose corresponding random variables are replaced by their expected values). To evaluate VSS, we have to solve the EEV problem, which exhibits the same structure as the stochastic programming model, but the corresponding first-stage decision variables are fixed according to the EV solution. Then $\mathrm{VSS}=\mathrm{EEV}-\mathrm{RP}$.

The main results in terms of costs and decision variables for the stochastic problem (RP) associated with the 16 cases are provided in Table 4 . Table 5 summarises the key values involved in EVPI and VSS.

Consistent with theoretical results, we found that WS $\leq \mathrm{RP} \leq \mathrm{EEV}$. Moreover, EVPI ranged from $356,542(0.5374 \%)$ to $148,153,582(18.97 \%)$, with a corresponding average value of $23,435,039(3.341 \%)$. Note that the average value is far from negligible, corresponding to $94 \%$ of the financial budget provided by the government. On the other hand, the VSS values are high in most cases; the average value found of 404,351,193 (66.28\%) indicates that it is possible to achieve significant gains by solving RP instead of $\mathrm{EV}$, which also justifies the use of the stochastic programming model.

The importance of prepositioning is evident. Without it (A1) EVPI and VSS decreases due to the high increase in backlogging costs compared to the base case (A0) that affects WS, RP and mostly EV. In this case, gathering perfect and accurate information about the random parameters would be more attractive than solving the stochastic model, since randomness plays a minor role in the problem. The stochastic solution without prepositioning presents a service level almost $4 \%$ worse with an increase of $22 \%$ in the objective function. Case A2 shows that prepositioning in all nodes has a negligible effect on EVPI and VSS values, since the WS, EEV and RP problems do not benefit much from prepositioning at $\mathrm{RC}$ nodes when prepositioning capacity is tight.

If some facilities were struck by a disaster so that $25 \%$ of depot inventory were lost (A3), then backlogging costs would increase, but without leading to deteriorated service levels. The result could be much worse if prepositioning in all nodes were permitted (A2). As the impact of the lost inventory is more pronounced in both RP and WS, but less evident in EEV, we found that EVPI slightly increases and VSS decreases accordingly.

Interestingly, EVPI increases dramatically without procurement (A4), but VSS drops more than $30 \%$. In fact, procurement does not improve the distribution of emergency emergency aid under perfect information, so WS does not vary significantly with or without procurement. However, this strategy is an important contingency decision for the stochastic problem. When it is not possible to purchase emergency aid in disaster areas, it is necessary to travel more among depots and warehouse in order to supply more demands. This in turn increases the first-stage costs of the fleet and the second-stage costs of the shipments. Due to the limited budget, the service level is then $3.45 \%$ worse. RP increases up to $21 \%$ in this case, approaching the upper bound value given by EEV. Thus RP-WS increases and EEV-RP decreases.

The effect of increasing supply and/or financial donations (A5, A6 and A7) in both EVPI and VSS is not clear due to the complex inter-relationship between these two random variables. A further increase in supply (A5) yields higher EVPI and VSS, but by assuming that an increase in budget that is sufficient to supply most demands (A6) decreases EVPI and VSS; similar results were found in Rennemo et al (2014). In the latter case (A6), uncertainty is reduced by increasing the investment in procurement by $30 \%$ and 
by improving fleet usage, instead of hiring a larger (possibly more expensive) fleet. Finally, EVPI and VSS experience a cross-effect when supply and donation increase simultaneously (A7): on the one hand, the improvement of service levels by almost $2.5 \%$ indicate that it is more likely to satisfy demand (the EVPI decreases); on the other hand, the EV solution leads to an underestimated fleet of vehicles that in turn increases substantially the backlogging costs of the EEV problem, thus increasing VSS.

When all arcs/routes are available (A8), the decision maker can contract a smaller fleet of vehicles to ship emergency aid, with fewer trucks and helicopters. This in turn helps to decrease perfect information. Conversely, with more damaged arcs (A9), more vehicles are required, mainly those that depend less on existing infrastructure; as a result, the EVPI increases. The availability of arcs has a minor impact on VSS. In both cases, service levels are only marginally affected.

Cases A10, A11, A12 and A13 suggest that it becomes more difficult to meet demand without one or more types of vehicle (A10, A11 and A12), particularly if trucks are not available in which case boats are the most used vehicle. Reducing the capacity of the trucks (A10) leads to a less efficient distribution of emergency aid, mostly in the RP, EV and EEV problems, since trucks present the best capacity/cost ratio among the vehicles. The result is a large increase in the value of perfect information, but a minor increase in the VSS. On the contrary, far fewer transport resources are used when truck capacity is larger (A13). This in turn decreases rental, shipping and backlogging costs. Using the same rationale as in case A10, we have smaller EVPI and VSS values. Without trucks and boats (A12), perfect information increases substantially more than in case A10, thus being impractical and adopting wait-and-see solutions instead of using stochastic ones. On the other hand, the stochastic solution value decreases up to $15 \%$. All the aforementioned behaviours are due to the great impact of a less diversified fleet on rental and backlogging costs, particularly in the RP problems. Finally, case A11 suggests that without using helicopters, backlogging slightly increases, but this effect has a minor impact on EVPI and VSS.

In the absence of lead times (A14), only trucks are used to ship emergency aid, since the one-day lead time for trucks in the first period enforced the utilization of the remaining modes of transportation. As a consequence, rental, shipping, procurement, and backlogging costs were reduced significantly, but inventory costs increased $150 \%$ in comparison to the base case. Most extra inventory in relief centers results from extra shipments due to efforts to increase transportation efficiency (fleet usage is about 98\%). The consequence is an increase in EVPI and VSS, thus indicating that the cost of uncertainty cannot be ignored.

Prioritising the delivery of the medical kits (A15) improves the service level for this emergency aid up to $8 \%$, but this provides a very low fleet efficiency. In this case, the objective values of all of problems WS, RP, EV and EEV may represent the "cost of urgency" of the medical kits. The relative EVPI and VSS values indicate not only the low impact of randomness in the problem, but also that a stochastic solution is less justified than in most of the remaining cases.

Finally, (A16) shows that, even though the probabilities do not change substantially the structure of the solution, the objective function is much worse for equiprobable scenarios, as the probability of the more pessimistic and expensive scenarios (moderate and major) increases from 0.24 to 0.45 . As a result, both EVPI and VSS increase. 
Cases A1, A3, A4, A9, A10, and A12 suggest that in a situation of scarce resources, there is a trend of increasing EVPI and/or decreasing VSS values. This apparent behaviour is particularly pronounced in the cases where prepositioning and procurement are not allowed (A1 and A4), without trucks (A10), and without both trucks and boats (A12). Considering that decision-making is often more complex (and expensive) in the presence of uncertainty, it is natural that scarcity impacts more in these situations than in situations under perfect information. Mathematically, we have an increase in RP greater than in WS, which intensifies EVPI. Due to the pre-determined first-stage decisions based on average values, the EEV problem is not as sensitive to resources shortage as RP, and then VSS tends to decrease.

[Insert Table 5 and Table 4 here]

Since the prepositioning capacity can be viewed as a strategic decision, specially useful in sudden/localised onset disasters (Apte and Yoho, 2011), and decision-makers may have few bases on which to decide its value in practical contexts, we perform a sensitivity analysis to show the impact of increasing it with respect to the main decision variables of the model. Table $\mathrm{J}$ in Appendix A.3 shows the detailed results. As expected, the objective value decreases as the amount of prepositioned stock increases due to the great reduction experienced by the level of overall unmet demand. However, the increase in prepositioning capacity has only a marginal effect in service levels, e.g., the double amount of prepositioned stock improves service levels in less than $1.7 \%$. In practice, it is not desirable to manage high amounts of prepositioned stock, as it may involve extra coordination among the different players of the humanitarian chain.

Increasing the prepositioned capacity also helps to improve the fleet usage via contracting a smaller fleet of vehicles in the first-stage, but using the overall vehicle capacity more efficiently in the secondstage. This strategy is partially responsible for the increase in the holding costs. Figure 6 illustrates these behaviours. Shipping and procurement costs do not present any particular trend as prepositioning stock increases.

\section{[Insert Figure 6 here]}

\subsection{Risk-Averse Results}

The proposed risk-averse models were analysed for $\phi=0.7$ and $u=0.9(\mathrm{CVaR})$, and $\phi=0.4(\mathrm{SD})$, by considering all the proposed instances A0-A16. Tables K and L in Appendix A.3 summarize the performance of the models. The results of all instances were enhanced by at least one risk approach in either unmet demand or second-stage costs at the expense of a minor "price" of risk aversion. In fact, with exception of the minimax approach that incurs a high price for less risky solutions in case A4 (when procurement is not allowed), the average price varies from $0.5 \%$ (SD) to almost $3 \%$ (minimax).

The semideviation model presents the lowest price of risk aversion, but the overall results are not very encouraging. In fact, its average and best performances across all the instances are clearly dominated by the CVaR model in terms of unmet demand and second-stage costs. On the other hand, CVaR provides the best improvement in unmet demand and second-stage costs in most instances. With CVaR, the distribution-aid plan is able to fulfill promptly 56,351 more emergency aid units than in the risk-neutral approach, approximately 1,400 more emergency aid units per scenario. Although this number sounds 
negligible in a commercial supply chain, since it represents less than $1 \%$ of total demand, it is sufficient to supply overall demand in affected areas with a lower number of victims, which is in accordance with the attitude of humanitarian logistics. CVaR also mitigates unmet demand in the worst-case scenario in 12 cases. On average, almost 5,000 more emergency aid units are delivered right-on-time in the most pessimistic scenario. This gain is substantially better in case A10 (without trucks), where the corresponding improvement is 52,000 emergency aid units. As a consequence of this better policy for demand fulfillment, overall costs associated with worst-case scenarios reduce accordingly, as well as the standard deviation of second-stage costs.

The minimax-regret model is the most conservative approach to deal with risk aversion. Besides the higher increase in objective values, the model still fails more often in improving both unmet demand and costs, and provides the worst overall performance. On average, expected service levels deteriorate $6 \%$, and unmet demand worsens in the worst-case scenario. However, in the instances where minimax succeed in improving unmet demand in the worst-case scenario, it achieves the best average performance and the best overall result: up to 91,000 extra emergency aid units are delivered in the worst-case scenario of instance A1.

Compared to the risk-neutral results, the overall trend of the risk-averse models is twofold: (i) increasing first-stage costs; and (ii) decreasing the standard deviation of the second-stage costs. In the first case, the increase is consequence of a larger fleet of vehicles, or a more expensive fleet composed of bulky vehicles, that in turn helps to decrease second-stage costs, as more emergency aid is promptly delivered, avoiding as much as possible shortage and holding costs. Given that shortages are much higher than holding costs, the consequence of enforcing risk aversion is decreasing unmet demand. This behaviour is more pronounced when resources are very limited, e.g., without prepositioning, trucks and/or boats, and less evident with excess of resources. Decreasing the cost standard deviation over the scenarios leads to more equity regarding unmet demands over scenarios and affected areas. The concept of equity or fairness in humanitarian operations is important because all affected areas (or a set of emergency aid) need to be fulfilled as equitably as possible.

In order to further analyse which risk approach provides fewer changes in the operational results, i.e., which one is the most "stable" from the relief operations standpoint, we evaluated the standard deviation (StDev), the coefficient of variation (CV), and the quartile coefficient of variation (QCV) for each type of cost (first-stage, second-stage, and expected total) obtained for each risk-averse approach in the 17 proposed cases. CV was determined as (standard deviation/mean), whereas QCV was calculated as (Q75 - Q25)/(Q75 + Q25), where Q25 and Q75 are the corresponding first and third quartiles of the sample. Figure 8 suggests that CVaR not only leads to overall lower statistics (StDev, CV, QCV), but also that it is not the worst approach in any of the cases analysed. These results indicate that CVaR is indeed the most stable method. On the other hand, minimax presents the least stable behaviour over the proposed instances. Interestingly, semideviation is particularly efficient to reduce the quartile coefficient of variation of the first-stage decisions. Basically, more stable solutions are also more reliable. It means that, for example, the fleet of vehicles provided by the CVaR framework has a higher chance to be feasible (or it is easier to make that decision feasible) under disruptions compared to the same decision provided 
by the other approaches.

[Include Figure 8 here]

\subsection{Computational Efficiency of the Two-Phase Approach}

The computational efficiency improvement of the two-phase procedure compared to the exact method over the 17 proposed cases for RN, minimax, CVaR and SD approaches is now discussed. Here, the exact method means solving the original model directly via CPLEX 12.5. Averages, medians, upper and lower quartiles and extreme points of the elapsed times (in seconds) for all approaches are shown in Figure 7. Table 6 summarizes the results of two metrics that analyse the overall quality of the heuristic procedure: (i) the relative difference between the objective value of the two-phase procedure and the (sub)optimal objective value (gap); (ii) the ratio between elapsed times (ratio). Tables $\mathrm{M}$ and $\mathrm{N}$ in Appendix A.3 present the detailed results.

It is clear from the plots that, as expected, minimax was the most time consuming risk-averse framework: over the 17 cases, elapsed times of the exact approach varied from a minimum of 4,000 s (seconds) to a maximum of 14,000 s. The risk-averse model SD exhibited the second worst performance. Despite presenting more variation in performance, the CVaR instances were solved $14 \%$ faster than the corresponding RN instances, on average. The results show that the computational efficiency resulting from the two-phase procedure is much better than when solving the original model directly. In fact, most heuristic solutions are very near to the optimal, and their corresponding elapsed times are at least 17 times faster than solving the original model directly. The plots reveal that the elapsed time of the heuristic is at most $500 \mathrm{~s}$, against 14,400 s for the exact method. The heuristic procedure also exhibits less variation in performance than the exact approach. In some instances, the two-phase approach improved the solution of the original model, which is possible because of the $1 \%$ relative gap at termination, meaning that exact solutions had not been verified as optimal. The improvement is particularly noticeable in the minimax results regarding instances A3, A7 and A13. In many minimax instances, though, the two-phase procedure fails to provide solutions with tighter gaps.

As expected, heuristic and exact solutions are similar in structure, since both optimization models (approximate and exact) exhibit basically the same set of constraints. The overestimation of the number of vehicles by using the sum of weight and volume of emergency aid, though, generated an average of $12 \%$ more vehicles contracted in the first-stage in comparison to the exact solution (over the 17 cases of the risk neutral model), in which $4.5 \%$ are trucks, $60 \%$ are boats, and $1 \%$ are helicopters. In terms of fleet usage, the results showed that the heuristic solution is $55 \%$ worse than the exact method. On the other hand, in terms of service levels, the difference between both solutions is less than $0.1 \%$ in favour of the heuristic ones. The results for the risk-averse models are similar, and for this reason, we omitted them. Table $\mathrm{O}$ and Figure 1 in Appendix A.3 present the detailed results regarding this comparison.

Further tests based on larger networks with 30 nodes and/or 100 scenarios evidence the strong performance of the heuristic procedure. Indeed, the exact method provides in 14,400 s an average gap of $85 \%$, whereas the heuristic procedure yields in $4,500 \mathrm{~s}$ an average gap of only $5.6 \%$. These figures were evaluated over a set of 15 randomly generated instances, whose details are provided in Table $\mathrm{P}$ in Appendix A.3. Even though the elapsed times of the heuristic method increase substantially for larger 

failed. Those results encourage the study of alternative heuristic methods to solve more efficiently the simplified model, perhaps without solving it directly via commercial optimization packages.

[Insert Table 6 here]

[Insert Figure 7 here]

Remark. The wait-and-see problems were solved in $767.5 \mathrm{~s}$ ( $\pm 960.8 \mathrm{~s})$ on average; best and worst elapsed times were 43.10 and $3,426 \mathrm{~s}$, respectively. For the EEV problems, we have an average of $315.3 \mathrm{~s}( \pm 944.8$ $\mathrm{s})$, and best/worst elapsed times of $50.60 \mathrm{~s}$ and 3,980 s, respectively. All EV problems were solved in less than $40 \mathrm{~s}$.

\section{Conclusions and Future Challenges}

Despite a growing concern to improve the distribution of aid in relief activities, it is still challenging to deal with all the particularities of the humanitarian supply chain. This paper thus aimed to contribute to the existing literature by developing a new two-stage stochastic network flow model under practical assumptions that are rarely considered in previous similar papers, such as the dynamic multiperiod nature of disaster relief operations, limited budgets, fleet sizing, and a variety of uncertain data. A set of scenarios was generated by combining a recent classification of the magnitudes of general disasters and historical data from the 2011 Brazilian megadisaster in the Serrana region in Rio de Janeiro state. We also devised a solution procedure to provide good-quality solutions within plausible elapsed times and found encouraging results. The model demonstrated that it can help plan and organise relief to provide good service levels in most scenarios, and how this depends on the type of disaster and resources. Tests on 16 variants of the base case scenario provided further insights and pointers to the impact of single-factor changes, for example, levels of prepositioning. Additional results suggested that it might be possible to improve equity (fairness) in distribution by enforcing risk aversion. We demonstrated that our heuristic has a good performance for both real and random instances.

As disasters are complex events, the solutions provided by the mathematical models are not meant to be implemented arbitrarily, but with the involvement, input and supervision of agency experts and local people, as well as, for example, geographic information systems (GIS). Thus, it would be useful to contact some Brazilian agencies to get feedback from experts regarding the usefulness of the proposed approach in terms of mathematical models and solutions. Such feedback would be used to refine the models and better understand the applicability of our framework. Our findings also bring up new open and challenging questions, for example, how to solve more efficiently the proposed mathematical models, and the impact of incorporating additional equity-based objectives in the stochastic model, and a social profit for meeting demands promptly. Also, the management of disaster relief fleets could be an interesting future work, for example, we could analyse the impact of the heterogeneity of the fleet of each type vehicle (e.g. trucks with different capacities). These sub-types would be interchangeable amongst themsleves, which would change the structure of the problem (Van Wassenhove and Pedraza Martinez, 2012). Lastly, extensions to multistage stochastic models deserve further efforts. 


\section{Appendix: Supplementary Material}

Supplementary data associated with the proposed instances can be found attached, as well as extra results mentioned in the computational experiments.

Acknowledgments: The first author is grateful for grants 2013/08303-2 and 2015/26453-7 from the São Paulo State Research Support Foundation (FAPESP) and grants 470154/2013-6 and 306237/2014-8 from the Brazilian National Council for Scientific and Technological Development (CNPq). Both authors thank the Royal Academy of Engineering, London, for their support via Newton Research Collaboration grant NRCP1516/1/54.

\section{References}

Ahmadi M, Seifi A, Tootooni B (2015) A humanitarian logistics model for disaster relief operation considering network failure and standard relief time: A case study on san francisco district. Transportation Research Part E: Logistics and Transportation Review 75:145 - 163

Altay N, Green WG (2006) OR/MS in disaster operations management. European Journal of Operational Research 175:475-493

Apte A (2009) Humanitarian logistics: A new field of research and action. Foundations and Trends in Technology, Information and Operations Management 3(1):1-100

Apte A, Yoho K (2011) Strategies for logistics in case of a natural disaster. In: 8th Annual Acquisition Research Symposium, Graduate School of Business \& Public Policy, Naval Postgraduate School, May 11-12, CA, USA

Arnell NW, Lloyd-Hughes B (2014) The global-scale impacts of climate change on water resources and flooding under new climate and socio-economic scenarios. Climatic Change 122(1-2):127-140

Balcik B, Beamon BM, Krejci CC, Muramatsu KM, Ramirez M (2010) Coordination in humanitarian relief chains: Practices, challenges and opportunities. International Journal of Production Economics $126(1): 22-34$

Barbarosoğlu G, Arda Y (2004) A two-stage stochastic programming framework for transportation planning in disaster response. Journal of the Operational Research Society 55:45-53

Birge JR, Louveaux F (1997) Introduction to stochastic programming. Springer, New York

Bozorgi-Amiri A, Jabalameli MS, Mirzapour Al-e-Hashem SMJ (2013) A multi-objective robust stochastic programming model for disaster relief logistics under uncertainty. OR Spectrum 35(4):905-933

Brasil (2012) Anuário brasileiro de desastres naturais: ano 2011. Ministério da Integração Nacional/ Secretaria Nacional de Defesa Civil/ Centro Nacional de Gerenciamento de Riscos e Desastres Brasília: CENAD (in Portuguese) p 80, URL http://www.drm.rj.gov.br/index.php/downloads/category/13-regioserrana 

Accessed in March 9th 2016

Brito Junior I, Leiras A, Yoshizaki H (2013) Stochastic optimization applied to the pre positioning of disaster relief supplies decisions in Brazil. In: 24th Annual Conference of the Production and Operations Management Society, pp 1-10

Caunhye AM, Nie X, Pokharel S (2012) Optimization models in emergency logistics: A literature review. Socio-Economic Planning Sciences 46(1):4-13

Centre for Research on the Epidemiology of Disasters (2014) EM-DAT - Emergency Events Database. http://www.em-dat.net. Accessed online 25 August 2014

Chang MS, Tseng YL, Chen JW (2007) A scenario planning approach for the flood emergency logistics preparation problem under uncertainty. Transportation Research Part E: Logistics and Transportation Review 43(6):737-754

Davis L, Samanlioglu F, Qu X, Root S (2013) Inventory planning and coordination in disaster relief efforts. International Journal of Production Economics 141(2):561-573

Döyen A, Aras N, Barbarosoğlu G (2012) A two-echelon stochastic facility location model for humanitarian relief logistics. Optimization Letters 6(6):1123-1145

DRM (2011) Departamento de Recursos Mineirais do Estado do Rio de Janeiro. Megadesastre da Serra (in Portuguese) URL http://www.drm.rj.gov.br/index.php/downloads/category/13-regio-serrana

Duran S, Gutierrez MA, Keskinocak P (2011) Pre-positioning of emergency items for care international. Interfaces 41(3):223-237

Eshghi K, Larson R (2008) Disasters: Lessons from the past 105 years. Disaster Prevention and Management 17:62-82

Franks S (2013) Reporting disasters: Famine, aid, politics and the media. Hurst Publishers

Fritz C, Mathewson J (1957) Convergent behavior: a disaster control problem. Special report for the committee on Disaster Studies Disaster Study 9:1-102

Galindo G, Batta R (2013) Review of recent developments in OR/MS research in disaster operations management. European Journal of Operational Research 230(2):201-211

Gonçalves P (2011) Balancing provision of relief and recovery with capacity building in humanitarian operations. Operations Management Research 4:39-50

Holguín-Veras J, Jaller M, Van Wassenhove LN, Pérez N, Wachtendorf T (2012) On the unique features of post-disaster humanitarian logistics. Journal of Operations Management 30(7):494-506

Hong X, Lejeune MA, Noyan N (2015) Stochastic network design for disaster preparedness. IIE Transactions $47(4): 329-357$ 
Jr EJL, Ballard KN, Song CH (2012) Pre-positioning hurricane supplies in a commercial supply chain. Socio-Economic Planning Sciences 46(4):291-305

Kelle P, Schneider H, Yi H (2014) Decision alternatives between expected cost minimization and worst case scenario in emergency supply-second revision. International Journal of Production Economics In press

Kouvelis P, Yu G (1997) Robust Discrete Optimization and its Applications. Kluwer Academic Publishers, Dordrecht

Kovács G, Spens K (2009) Identifying challenges in humanitarian logistics. International Journal of Physical Distribution \& Logistics Management 39(6):506-528

Krokhmal P, Zabarankin M, Uryasev S (2011) Modeling and optimization of risk. Surveys in Operations Research and Management Science 16(2):49-66

Leiras A, de Brito Jr I, Queiroz Peres E, Rejane Bertazzo T, Tsugunobu Yoshida Yoshizaki H (2014) Literature review of humanitarian logistics research: trends and challenges. Journal of Humanitarian Logistics and Supply Chain Management 4(1):95-130

Li L, Jin M, Zhang L (2011) Sheltering network planning and management with a case in the gulf coast region. International Journal of Production Economics 131(2):431-440

Lin YH, Batta R, Rogerson PA, Blatt A, Flanigan M (2012) Location of temporary depots to facilitate relief operations after an earthquake. Socio-Economic Planning Sciences 46(2):112 - 123, modeling Public Sector Facility Location Problems

Markert A, Schultz R (2005) On deviation measures in stochastic integer programming. Operations Research Letters 33(5):441 - 449

Mete HO, Zabinsky ZB (2010) Stochastic optimization of medical supply location and distribution in disaster management. International Journal of Production Economics 126(1):76-84

Mulvey J, Vanderbei R, Zenios S (1995) Robust optimization of large-scale systems. Operations Research $43: 264-281$

Noyan N (2012) Risk-averse two-stage stochastic programming with an application to disaster management. Computers \& Operations Research 39(3):541 - 559

Noyan N, Balcik B, Atakan S (2015) A stochastic optimization model for designing last mile relief networks. Transportation Science DOI 10.1287/trsc.2015.0621, URL http://dx.doi.org/10.1287/trsc.2015.0621

Ogryczak W, Ruszczyński A (2001) On consistency of stochastic dominance and mean-semideviation models. Mathematical Programming 89:217-232 
Ortuño MT, Cristóbal P, Ferrer JM, Martín-Campo FJ, Muñoz S, Tirado G, Vitoriano B (2013) Decision aid models and systems for humanitarian logistics. a survey. In: Vitoriano B, Montero J (eds) Decision Analysis Aid Models for Disaster Management and Emergencies, Atlantis Computational Intelligence Systems, Series Volume 7, chap 2, pp 17-45

Oztaysi B, Behret H, Kabak O, Sari IU, Kahraman C (2013) Fuzzy inference systems for disaster response. In: Vitoriano B, Montero J, Ruan D (eds) Decision Aid Models for Disaster Management and Emergencies, Atlantis Computational Intelligence Systems, vol 7, Atlantis Press, pp 75-94

Peixoto Filho GEC (2013) Preparação e resposta a desastres no território nacional. In: Seminário Estadual Sobre Emergência Ambiental, Minas Gerais (presentation). Available in: $<$ http://www.meioambiente.mg.gov.br/images/stories/emergencia_ambiental/P2R2/seminarios/1getulio-costa-cenad.pdf $>$. Accessed in March 9th 2016

Presidency of the Republic, Brazilian Government (2013) Defesa civil terá centros com estoques estratégicos de material para atender pessoas atingidas por desastres. http://bit.ly/1AsYFTN. Accessed online 18 August 2014

Rawls CG, Turnquist MA (2010) Pre-positioning of emergency supplies for disaster response. Transportation Research Part B: Methodological 44(4):521-534

Rawls CG, Turnquist MA (2012) Pre-positioning and dynamic delivery planning for short-term response following a natural disaster. Socio-Economic Planning Sciences 46(1):46-54

Rennemo SJ, Rø KF, Hvattum LM, Tirado G (2014) A three-stage stochastic facility routing model for disaster response planning. Transportation Research Part E: Logistics and Transportation Review 62:116-135

Riis M, Andersen KA (2005) Applying the minimax criterion in stochastic recourse programs. European Journal of Operational Research 165(3):569-584

Rottkemper B, Fischer K, Blecken A (2012) A transshipment model for distribution and inventory relocation under uncertainty in humanitarian operations. Socio-Economic Planning Sciences 46(1):98-109

Salmerón J, Apte A (2010) Stochastic optimization for natural disaster asset prepositioning. Production and Operations Management 19(5):561-574

dos Santos LGF (2013) Ações da SEDEC/CBMERJ Região Serrana - 2011/2013 (presentation). Available in: <http://www2.camara.leg.br/atividade-legislativa/comissoes/comissoestemporarias/externas/54a-legislatura/desastres-na-regiao-serrana-do-rio-de-janeiro/audienciaspublicas-1/apresentacao-do-cel-luis-guilherme-ferreira-dos-santos-defesa-civil-rj-1/apresentacao-docel-luis-guilherme-ferreira-dos-santos-defesa-civil-rj>. Accessed in March 9th 2016

Sarkis J, Spens KM, Kovács G (2010) A study of barriers to greening the relief supply shain. In: Kovács G, Spens KM (eds) Relief supply chain for disasters: humanitarian aid and emergency logistics, IGI Global, Hershey, PA, pp 196-207 

14(4):1237-1249

Sheu JB, Pan C (2015) Relief supply collaboration for emergency logistics responses to large-scale disasters. Transportmetrica A: Transport Science 11(3):210-242

Smith E, Wasiak J, Sen A, Archer F, Burkle FM (2009) Three decades of disasters: A review of disasterspecific literature from 1977-2009. Prehospital and Disaster Medicine 24:306-311

De la Torre LE, Dolinskaya IS, Smilowitz KR (2012) Disaster relief routing: Integrating research and practice. Socio-Economic Planning Sciences 46(1):88-97, DOI 10.1016/j.seps.2011.06.001

Toyasaki F, Wakolbinger T (2011) Impacts of earmarked private donations for disaster fundraising. Annals of Operations Research pp 1-21

Valencio N (2014) Desastre como prática sociopolótica de solapamento da segurança humana. In: do Carmo R, Valencio N (eds) Segurança humana no contexto dos desastres (in portuguese), RiMa, São Carlos, Brazil, pp 15-44

Van Wassenhove LN (2006) Humanitarian aid logistics: supply chain management in high gear. Journal of the Operational Research Society 57(5):475-498

Van Wassenhove LN, Pedraza Martinez AJ (2012) Using OR to adapt supply chain management best practices to humanitarian logistics. International Transactions in Operational Research 19(1-2):307-322

Yodmani S (2001) Disaster risk management and vulnerability reduction: Protecting the poor. Paper presented at the Asia Pacific Forum on Poverty organized by the Asia Development Bank, Asian Development Bank, http://http://www.adpc.net/infores/adpc-documents/povertypaper.pdf, accessed in 26 January 2015

Zagefka H, Noor M, Brown R, de Moura GR, Hopthrow T (2011) Donating to disaster victims: Responses to natural and humanly caused events. European Journal of Social Psychology 41(3):353-363

Özdamar L, Ertem MA (2015) Models, solutions and enabling technologies in humanitarian logistics. European Journal of Operational Research 244(1):55 - 65

Özdamar L, Ekinci E, Küçükyazici B (2004) Emergency logistics planning in natural disasters. Annals of Operations Research 129(1-4):217-245 


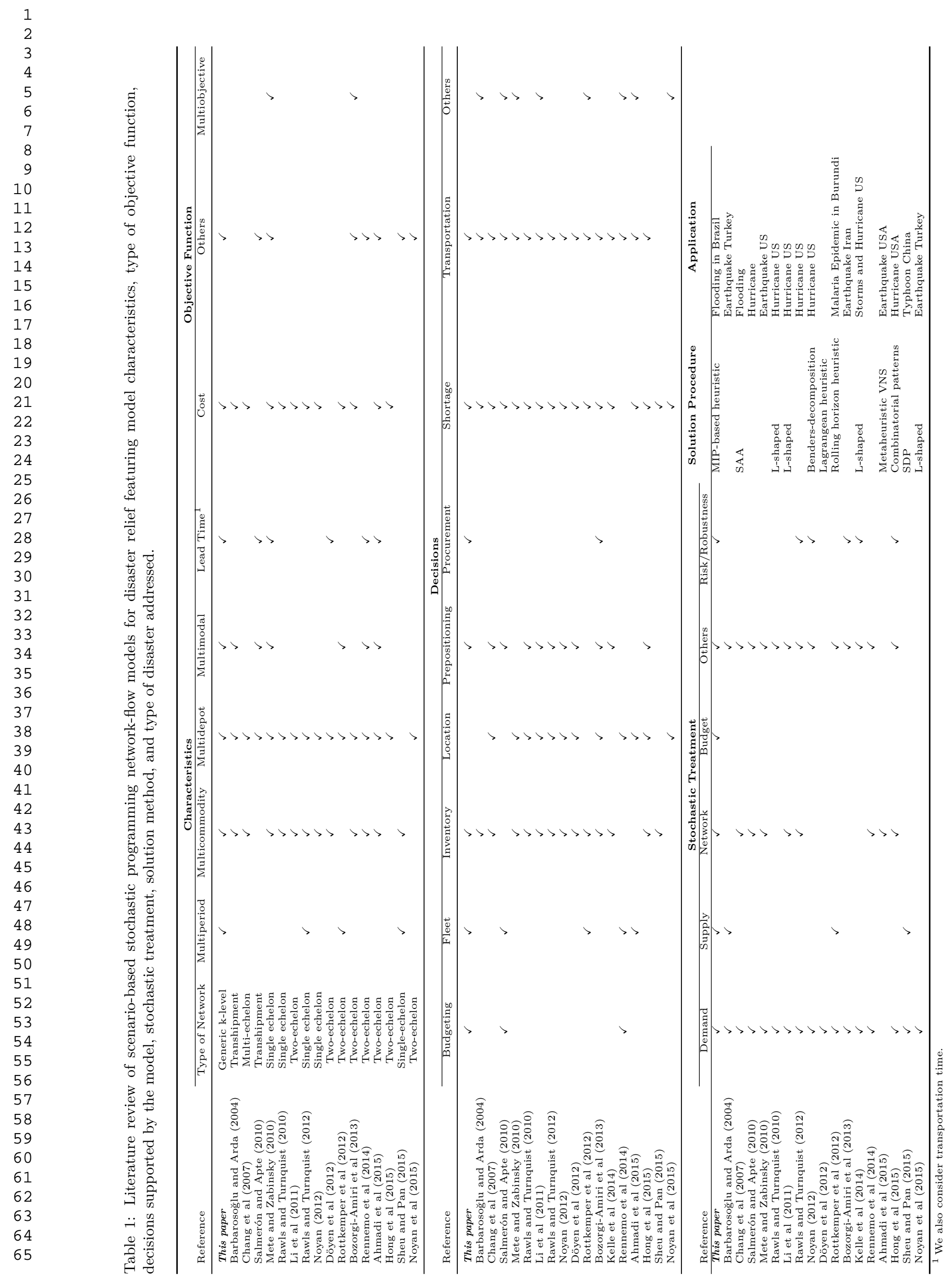


Table 2: Overview of the proposed risk-averse models in terms of necessary inputs and outputs.

\begin{tabular}{lccccr}
\hline \multirow{2}{*}{ Model } & \multicolumn{4}{c}{ Inputs } & \\
\cline { 2 - 5 } & $\begin{array}{c}\text { Probability } \\
\text { of scenarios } \\
\left(\pi^{\xi}\right)\end{array}$ & $\begin{array}{c}\text { Wait-and-see } \\
\text { solutions } \\
\left(W^{\star}(\xi)\right)\end{array}$ & $\begin{array}{c}\text { Risk } \\
\text { parameter } \\
(\phi)\end{array}$ & $\begin{array}{c}\text { Confidence } \\
\text { level } \\
(u)\end{array}$ & Outputs \\
\hline F2: Minmax & & $\checkmark$ & & $\checkmark$ & Maxium regret $(\Phi)$ \\
F3: CVaR & $\checkmark$ & & $\checkmark$ & VaR, CVaR, $\Theta^{\xi}$ \\
F4: SD & $\checkmark$ & & $\checkmark$ & Semideviation $\left(\Delta^{\xi}\right)$ \\
\hline
\end{tabular}

Table 3: Required emergency aid for survivors of a disaster and the characteristics of each type of emergency aid.

\begin{tabular}{llllll}
\hline $\begin{array}{l}\text { emergency aid } \\
\text { Type }\end{array}$ & $\begin{array}{l}\text { Weight } \\
\text { in kg }\end{array}$ & $\begin{array}{l}\text { Volume } \\
\text { in l }\end{array}$ & $\begin{array}{l}\text { Quantity per } \\
\text { day per victim }\end{array}$ & $\begin{array}{l}\text { Procurement } \\
\text { price }^{1}\end{array}$ & $\begin{array}{l}\text { Prepositioning } \\
\text { capacity in units }\end{array}$ \\
\hline Food & 23 & 30 & $1 / 5 \mathrm{kit}$ & 86.18 & 20,000 \\
Water & 20 & 20 & $5 \mathrm{l}$ & 7.50 & 19,000 \\
Hygiene & 3 & 10 & $1 \mathrm{kit}$ & 27.03 & 10,328 \\
Cleaning & 7 & 16 & $1 / 5 \mathrm{kit}$ & 60.27 & 1,000 \\
Mattress & 2 & 103 & 1 unit & 29.90 & 30,611 \\
Medicine & 14 & 11 & $1 / 90 \mathrm{kit}$ & 85.20 & 0 \\
\hline
\end{tabular}

${ }^{1}$ All costs are given in Brazilian Real (BRL). 


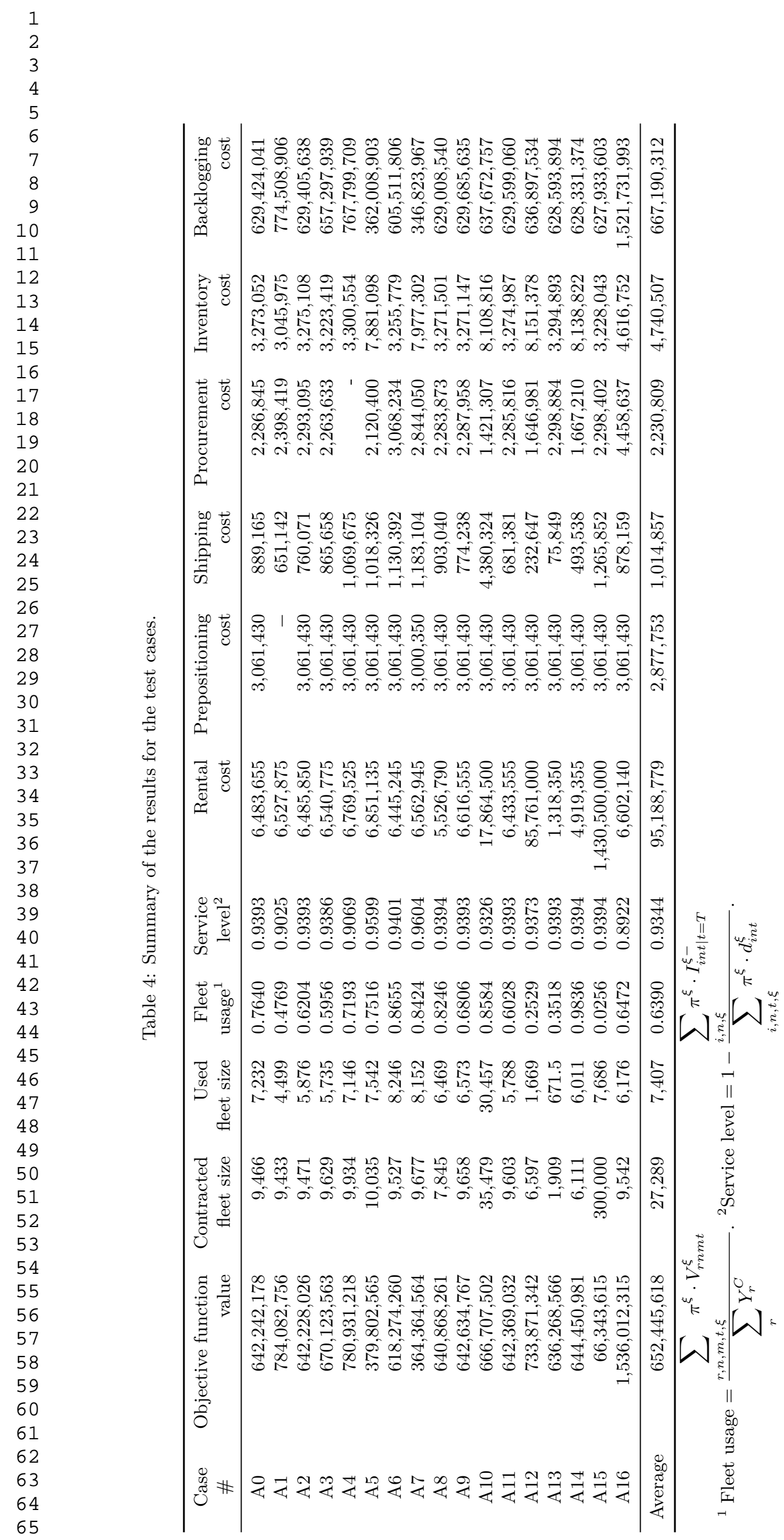




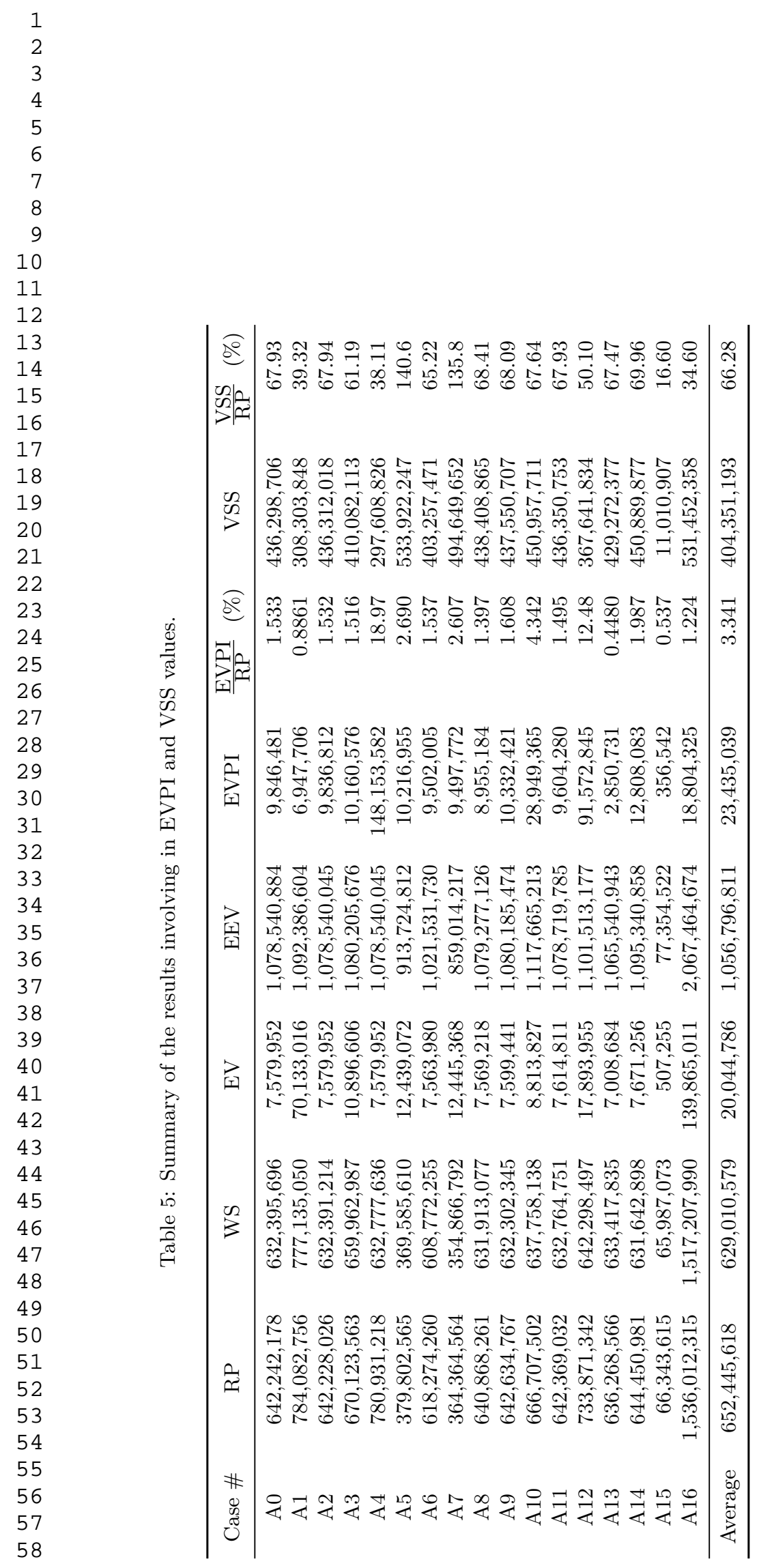


Table 6: Comparison between computational efficiency of the exact and the two-phase approach over the propose instances.

\begin{tabular}{cllllllll}
\hline & \multicolumn{3}{c}{ RN } & \multicolumn{2}{c}{ MinMax } & \multicolumn{2}{c}{ CVaR } & \multicolumn{2}{c}{ SD } \\
\cline { 2 - 8 } Case & Gap (\%) & Ratio & Gap (\%) & Ratio & Gap (\%) & Ratio & Gap (\%) & Ratio \\
\hline A0 & 0.2987 & 29.14 & 36.99 & 44.80 & 0.0126 & 24.26 & 0.2065 & 26.11 \\
A1 & 0.1198 & 9.535 & 29.61 & 70.06 & 0.0216 & 4.454 & 0.0900 & 19.81 \\
A2 & 0.3009 & 21.77 & 34.30 & 50.03 & 0.0127 & 24.95 & 0.1866 & 35.35 \\
A3 & 0.3457 & 9.856 & -6.003 & 64.04 & 0.0330 & 10.00 & 0.2180 & 16.76 \\
A4 & 0.2543 & 22.60 & 0.1925 & 37.16 & 0.0323 & 28.61 & 0.4757 & 23.09 \\
A5 & 0.4982 & 19.91 & 11.35 & 34.70 & 0.0518 & 20.44 & 0.3996 & 20.97 \\
A6 & 0.3372 & 20.30 & 12.59 & 37.15 & 0.0171 & 16.49 & 0.2690 & 29.18 \\
A7 & 0.4792 & 11.60 & -20.93 & 28.53 & 0.0403 & 14.11 & 0.4508 & 16.50 \\
A8 & 0.0931 & 32.36 & 10.09 & 46.96 & 0.0452 & 8.214 & 0.0495 & 19.57 \\
A9 & 0.3888 & 19.98 & 29.92 & 55.47 & -0.0081 & 23.84 & 0.2466 & 19.10 \\
A10 & 2.074 & 10.29 & 10.26 & 67.43 & 0.0852 & 8.384 & 0.5322 & 21.62 \\
A11 & 0.1109 & 43.66 & 122.9 & 146.4 & 0.0568 & 21.92 & 0.2067 & 50.46 \\
A12 & 0.2925 & 36.15 & 2.297 & 59.99 & -0.0021 & 4.483 & 0.2611 & 29.75 \\
A13 & -0.0365 & 12.83 & -67.52 & 41.38 & -0.2581 & 21.29 & -0.4524 & 23.36 \\
A14 & 0.5014 & 9.150 & 25.94 & 57.57 & -0.0076 & 11.91 & 0.2016 & 27.12 \\
A15 & -0.0683 & 61.90 & 100.0 & 39.68 & -0.0107 & 39.32 & -0.0403 & 44.99 \\
A16 & 0.1514 & 7.808 & 9.304 & 47.86 & 0.0067 & 9.101 & 0.0258 & 39.82 \\
\hline Average & 0.3613 & 22.28 & 20.07 & 54.66 & 0.0076 & 17.16 & 0.1957 & 27.27 \\
\hline
\end{tabular}

${ }^{1} \mathrm{Gap}=\frac{Z^{H}-Z^{M I P}}{Z^{M I P}}$, where $Z^{H}$ refers to the objective value of the two-phase procedure, and $Z^{M I P}$ is the objective value of the exact approach.

${ }^{2}$ Ratio $=\frac{\text { elapsed }^{M I P}}{\text { elapsed }^{H}}$, where elapsed ${ }^{H}$ is the elapsed time of the two-phase procedure, and elapsed $^{M I P}$ is the elapsed time of the exact approach.

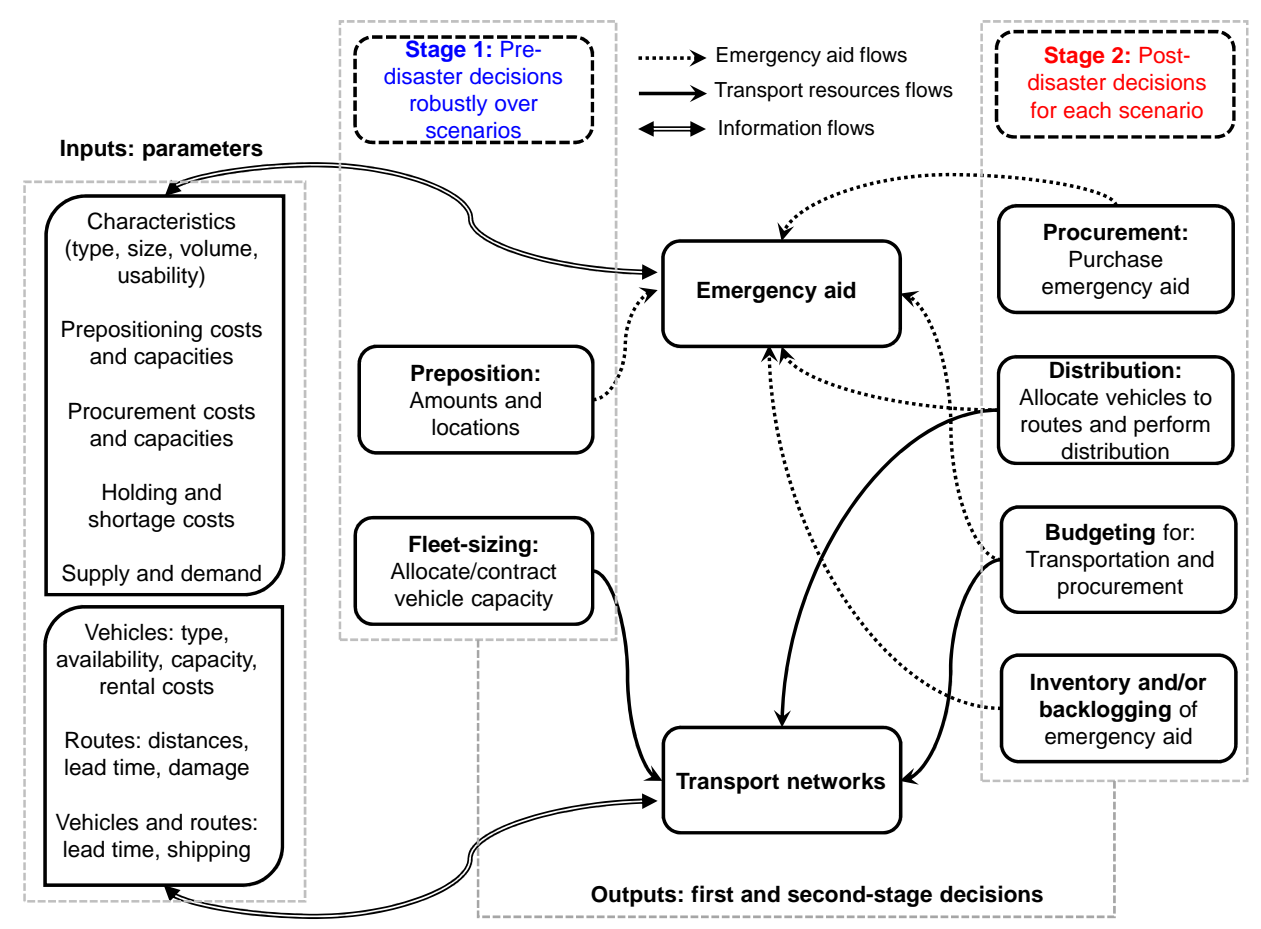

Figure 1: Overview of the model's structure and usage. 


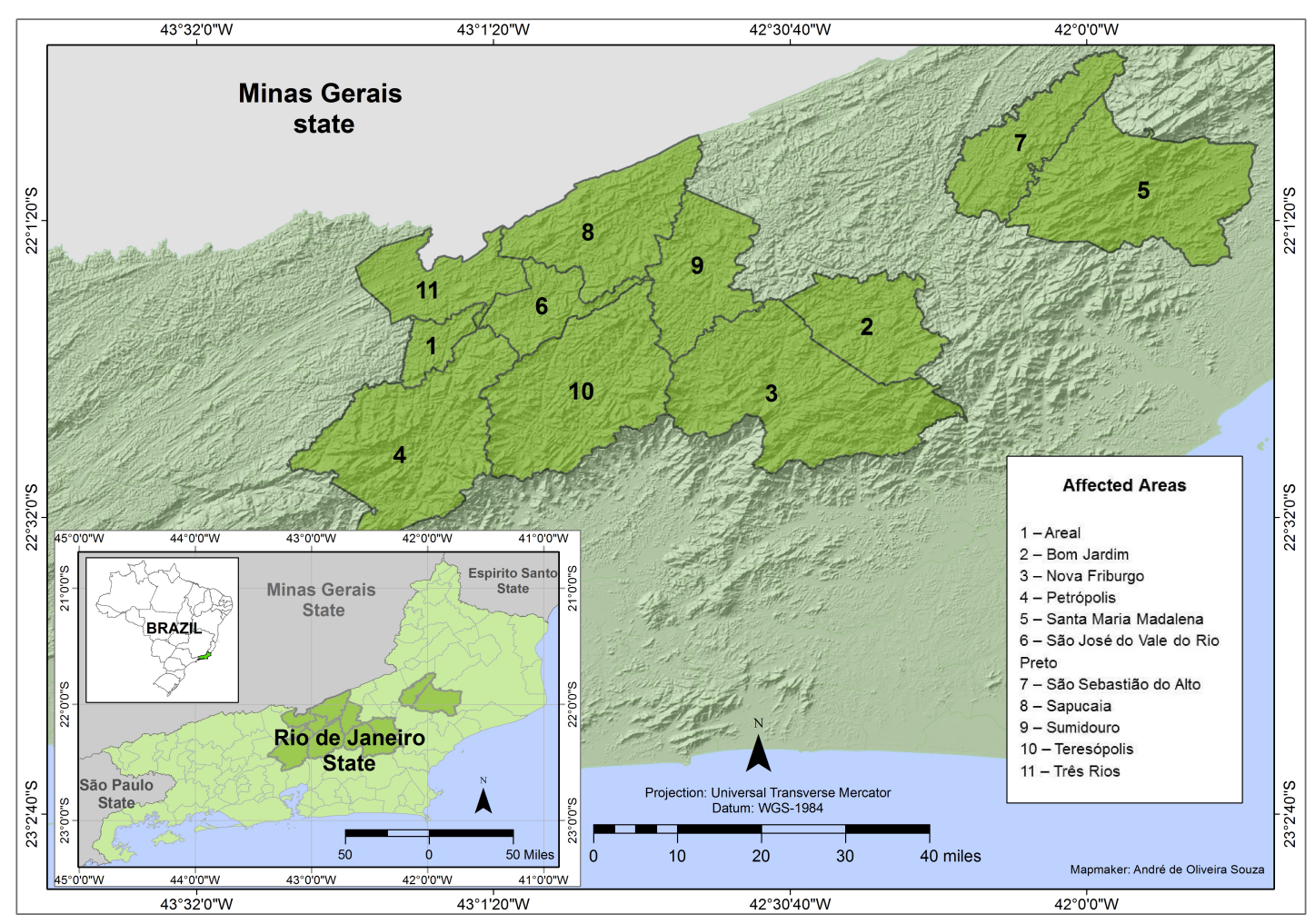

Figure 3: Map showing the Serrana region of Rio de Janeiro state. 


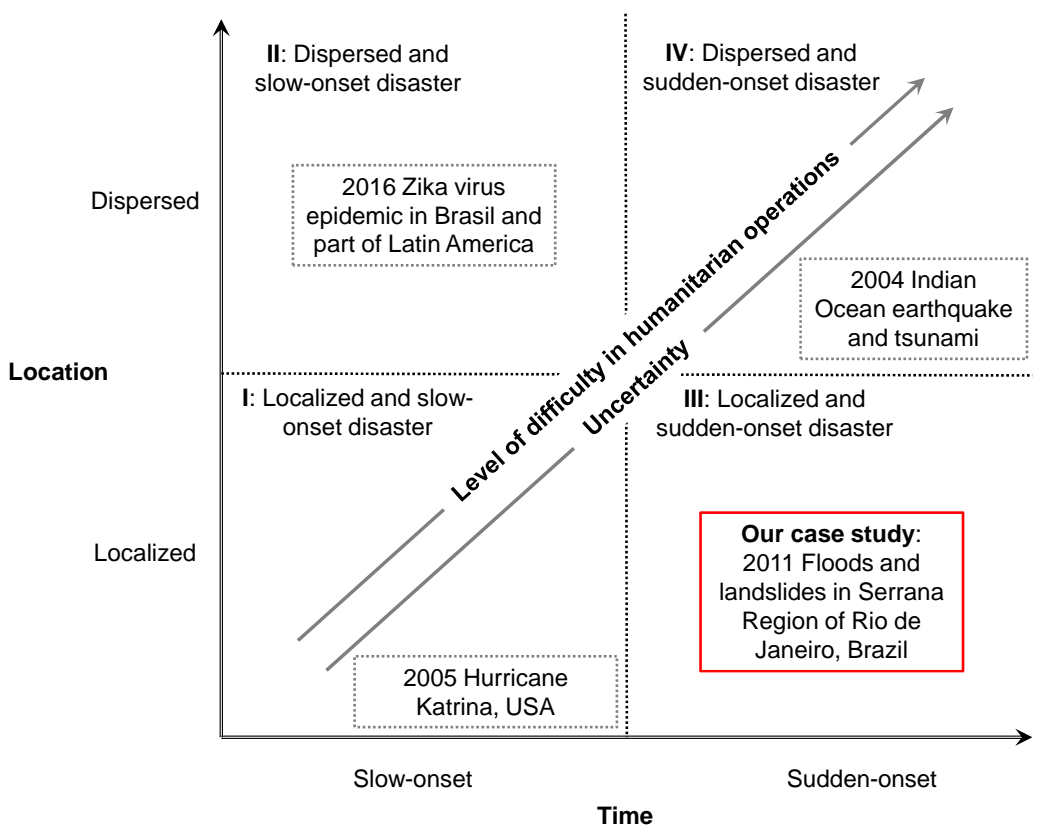

Figure 4: Disaster categorisation proposed by Apte (2009). Adapted from Apte and Yoho (2011); Apte (2009). 


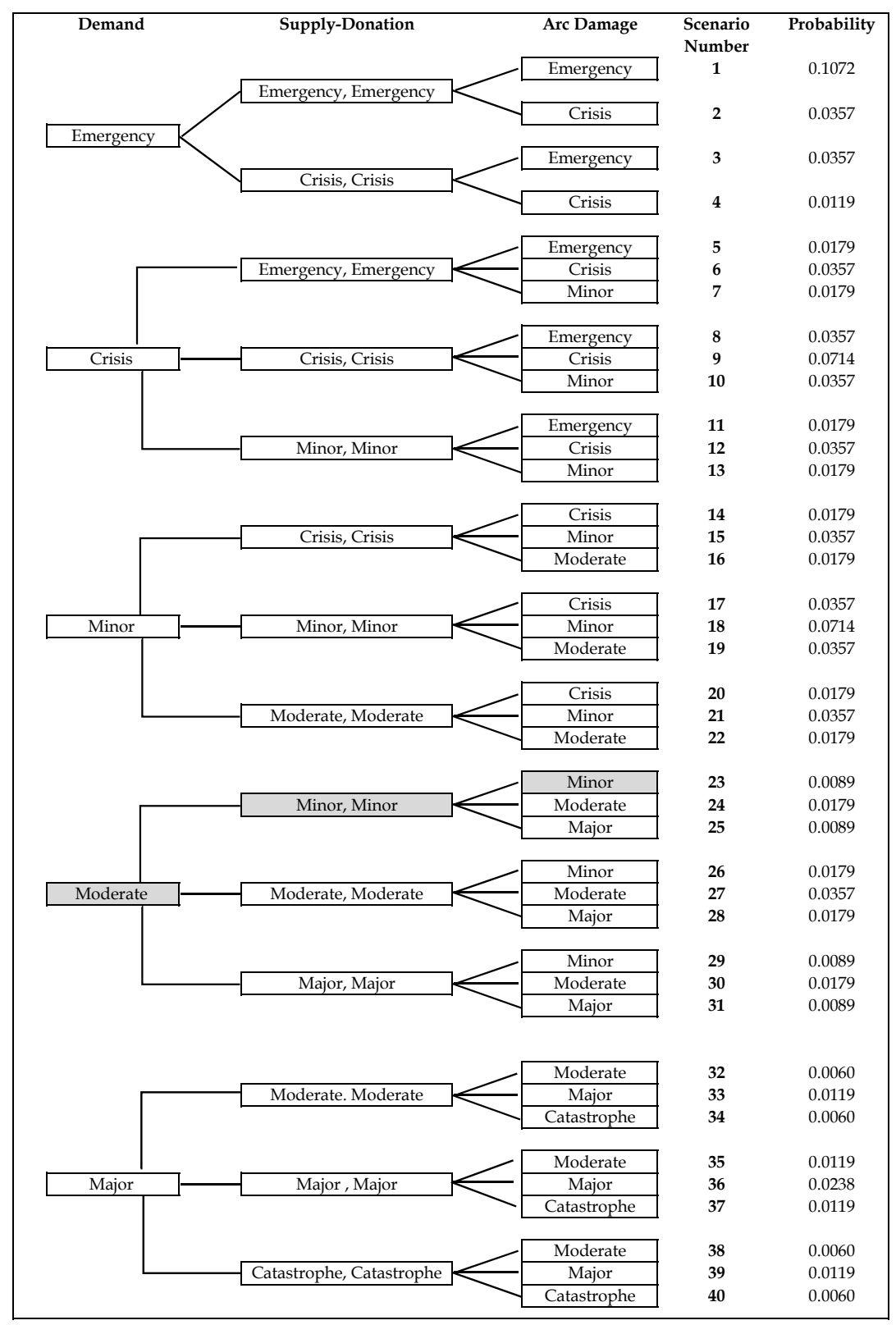

Figure 5: Set of scenarios as the combination of the four stochastic parameters, totaling 40 scenarios. 


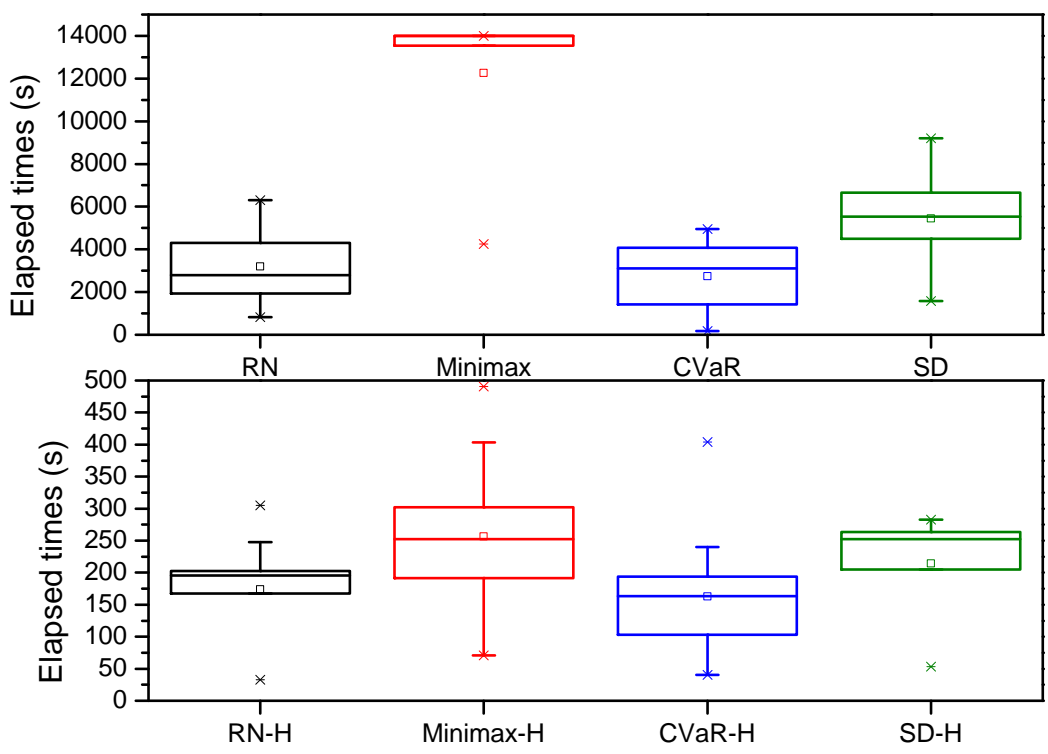

Figure 7: Elapsed times of all instances regarding the exact approach (above) and the two-phase heuristic approach (below). Box-plots represent, in this order, maximum values, 95th percentiles, 75th percentiles, medians, means, 25th percentiles, 5th percentiles, and minimum values. 

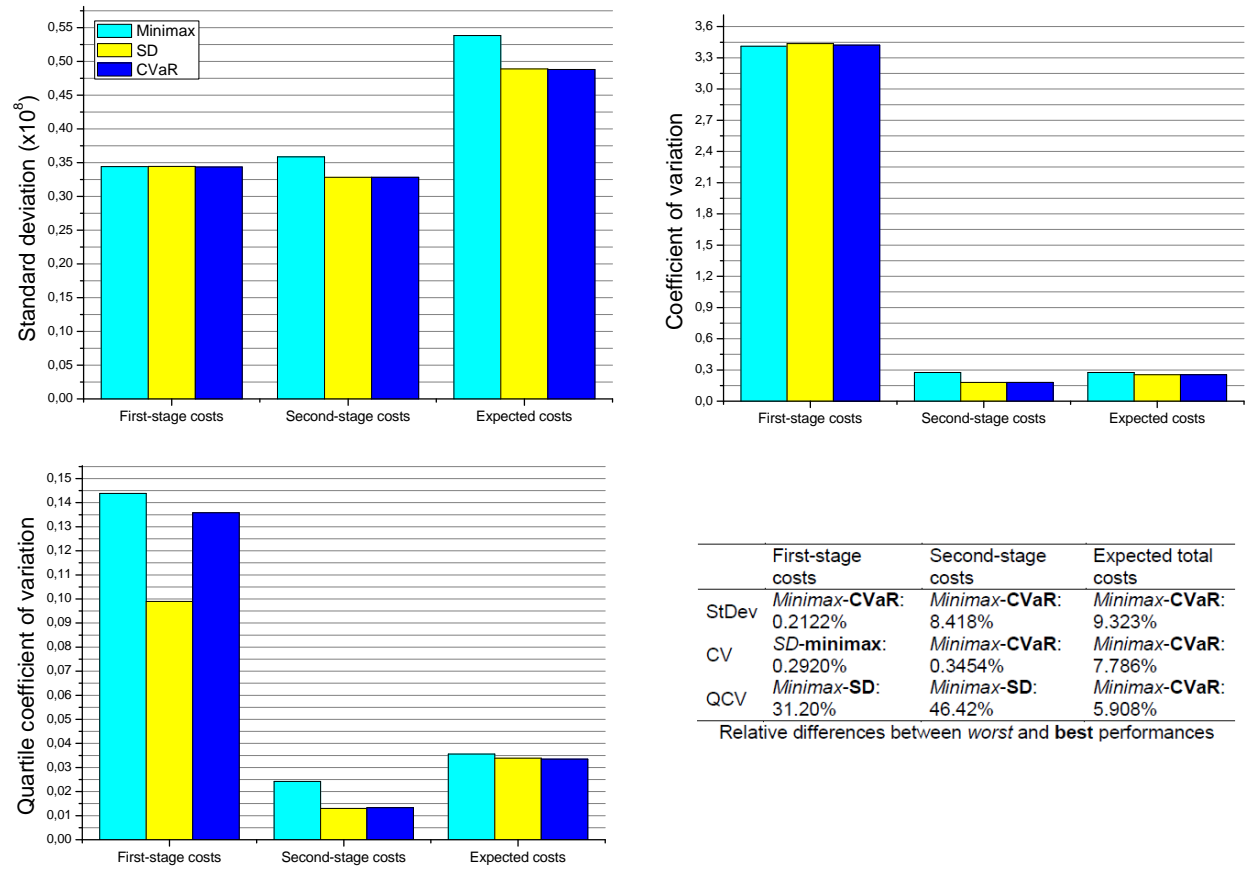

Figure 8: Stability analysis of the costs over the 17 proposed instances according to each risk-averse approach. The bars illustrate the following statistics: standard deviation, coefficient of variation evaluated as standard deviation/mean, and quartile coefficient of variation, evaluated as (Q75-Q25)/(Q75+Q25), where Q25 and Q75 are the corresponding first and third quartiles of the sample. 